FR-PHENO-2015-011

\title{
Two-loop Integrand Decomposition Into Master Integrals And Surface Terms
}

\author{
Harald Ita \\ Institut of Physics, University of Freiburg \\ D-79104 Freiburg, Germany
}

\begin{abstract}
Loop amplitudes are conveniently expressed in terms of master integrals whose coefficients carry the process dependent information. Similarly before integration, the loop integrands may be expressed as a linear combination of propagator products with universal numerator-tensors. Such a decomposition is an important input for the numerical unitarity approach, which constructs integrand coefficients from on-shell tree amplitudes. We present a new method to organize multi-loop integrands into a direct sum of terms that integrate to zero (surface terms) and remaining master integrands. This decomposition facilitates a general, numerical unitarity approach for multiloop amplitudes circumventing analytic integral reduction. Vanishing integrals are well known as integration-by-parts identities. Our construction can be viewed as an explicit construction of a complete set of integration-by-parts identities excluding doubled propagators. Interestingly, a class of 'horizontal' identities is singled out which hold as well for altered propagator powers.

PACS numbers: 11.15.Bt, 11.25.Db, 11.55.-m, 12.38.-t, 12.38.Bx
\end{abstract}




\section{INTRODUCTION}

Currently the experiments at the Large Hadron Collider (LHC) are entering a new energy and luminosity regime. Further upgrades are expected for a number of years to come. The increasing amount of data will allow to zoom into known physics and extend the discovery potential for new physics. An important ingredient in this quest are precise predictions which match the measurements' standards. Predictions for key observables will be necessary, but providing a larger set of predictions beyond this minimal set will be a clear benefit. Here we present new theoretical methods towards these latter aims.

An important input for precision predictions are first-principle computations in perturbative quantum-field theory. In recent years, significant progress has been made by the theory community in providing predictions through automated fixed-order computations including quantum corrections [8-15]. These have already lead to a wide class of next-to-leading order (NLO) predictions for Standard Model processes. In addition, a number of impressive two-to-two next-to-next-to-leading order (NNLO) results [1 [ 6] $]$ and further higher-order predictions [7] have become available. These developments have been driven by a combination of analytic and numerical advances for computing loop integrals. At one-loop level one can highlight explicit [16] and implicit methods [9, 17-22] for reducing (tensor) loop integrals to a standard set of master integrals. Similarly, at two-loop level, explicit analytic reduction techniques for integrals [23 26, 28, 29] play an important role. Here we discuss methods which bypass analytic integral reduction and make a numerical approach to multi-loop computations possible. Such methods are at the core of the unitarity based approaches [9 13 , 30] to NLO predictions and allowed to push towards processes with many partons [31-35]. We are motivated by these results to explore a numerical unitarity approach for multi-loop amplitudes.

The unitarity method [17, 18, 36, 37] has continuously provided cutting edge results for formal as well as phenomenology oriented multi-loop amplitudes (see e.g. [38 40] and [36, 41]). This method relates universal representations of amplitudes in terms of master integrals to full scattering amplitudes. By comparing the analytic structure (e.g. branch cuts) of both representations the process dependent coefficients of master integrals are obtained. In this approach, the cutting operation simplifies the loop integrals to phase-space integrals over onshell tree-level input. On the one hand, the strength of this approach arises from efficiently 
dealing with physical (on-shell) building blocks. On the other hand, the unitarity approach provides an implicit integral-reduction mechanism, since by cutting one targets coefficients of master integrals very directly.

In this article we discuss a numerical variant of the unitarity approach. This approach is well developed at one-loop level [9, 21, 22] and we extend it to higher-loop orders. In the numerical approach, the loop integrations are delayed to the very end of the computation. First one compares the rational integrands of the Feynman amplitudes with a universal basis of loop integrands. Delaying the loop integration, however, comes with a price; in order to maintain the equality of the integrand basis and Feynman amplitudes additional terms, i.e. surface terms, have to be added to the basis. These are terms that integrate to zero eventually but are required in intermediate steps. The explicit construction of the multi-loop surface terms is the main result of this article. The importance of the surface terms becomes clear in the remaining computational steps. Once the coefficients of the integrand basis have been obtained (through solving linear equations), the loop integration is performed. In this final step surface terms can be dropped and only the master integrals have to be provided to yield the loop amplitudes. In this way the reduction of tensor integrals is accomplished by the integrand parametrization and is implicit. Given the classification of surface terms we obtain a general, i.e. process and multiplicity independent, numerical algorithm.

Even though the unitarity method operates on-shell, the surface terms have to be known off-shell. This is required, since already computed unitarity cuts have to be subtracted in cuts with fewer on-shell propagators in order to avoid double counting. However, the cut conditions can only be relaxed if we have a way to take results off-shell. The prescription to go off-shell is provided through the integrand parametrization.

A number of recent developments have advanced the unitarity method to a promising approach for automated multi-loop computations in QCD. Parametrizations of loop integrands have been developed recently [42 44], which are given in terms of a minimal basis of irreducible integrands. These parametrizations identify a subset of the terms (spurious numerators) which integrate to zero, but not all. Thus, standard reduction techniques [2326, 29] are required to obtain a final representation in terms of master integrals. Here we put forward a different type of representation of loop integrands which is organized into surface terms and master integrals. In our approach, the integral reduction is built-in and does not have to be performed in a second step, which is important in a numerical approach. Fur- 
thermore, in analogy to the numerical one-loop unitarity approach, the integral coefficients can be computed through generalized unitarity cuts [45] by solving linear equations (e.g. via Fourier transforms). This approach does not require integration over multi-dimensional phase spaces and thus differs from the direct extraction of integral coefficients [46] or possible extensions using the duality between master integrals and homology cycles of the phase spaces [47, 48, 52]. Nevertheless, the latter approaches hold the promise to be very efficient once available in a complete way.

Technically, a number of new observations lead to the present construction. First of all, we combine integration-by-parts (IBP) identities and master integrals to parametrize the loop integrands. Although this approach is natural it has not been appreciated for the numerical unitarity approach so far. For the unitarity approach it is best to focus on IBP identities that do not involve integral topologies with doubled propagators [49]. The identities are obtained from a specialized set of vector fields in loop-momentum space. We provide the explicit form of such IBP vectors. Algorithms to obtain IBP vectors have been suggested in the original literature and improved in refs. [50, 51]. We give a complete set of (off-shell) vectors for two-loop topologies. This construction is important in order to obtain compact analytic expressions as well as numerical control throughout momentum space. The presented construction reproduces the known results at one-loop level [22].

Moreover, we find interesting properties of the IBP vectors; using general coordinate transformations to adjust the integration variables to the integral topology, IBP vectors can be constructed explicitly with pen and paper. In fact, the IBP vectors turn out to come in two types, (complexified) rotations in momentum space, which leave propagators invariant, and scaling transformations of the propagators. For our construction the former 'horizontal' generators will be most important. A special example of such horizontal vectors was given already in [49] (based on Gram determinants).

Although we construct special IBP relations that do not double propagators, we obtain a much bigger set of IBP relations. In fact, once the numerators of the horizontal IBP relations are obtained, the propagator powers may be changed to give new relations.

We observe that the IBP vectors are tangent vectors to the unitarity-cut phase spaces. This property allows one to link off-shell and on-shell information on unitarity cuts; we show (see section $\llbracket$ IIG ) that surface terms from special IBP relations are as well surface terms on the unitarity-cut phase spaces. Interestingly, we observe as well a Lie-algebra 
structure which simplifies the construction of the IBP vectors. This structure appears to be fundamental linking unitarity cuts to full amplitudes (section IVF).

Finally, the link between off-shell and on-shell information allows one to relate master integrands and surface terms to closed and exact holomorphic forms on the phase spaces, respectively. The number of master integrals is then given through topological properties of the unitarity-cut phase spaces, that is the number of closed-modulo-exact forms. The importance of cohomology for the construction of on-shell IBP identities has been discussed recently [48]. We setup a related but simplified on-shell approach to multi-dimensional phase spaces. Although we construct off-shell surface terms, the on-shell perspective serves as valuable guidance and a cross check. In fact, the on-shell construction is simpler and its completeness can be verified in a combinatorial way. We use the on-shell approach to verify that the constructed surface terms are complete.

Here we focus on planar two-loop integrals, however, our methods can be extended to nonplanar as well as a full $D$-dimensional approach, and we suggest how the generalizations can be done. In fact, we give IBP vectors for the non-planar two-loop topologies. Furthermore, we work with generic non-vanishing internal and external masses and, thus, capturing much of the $D$-dimensional aspects. We believe that the IBP vectors are sufficient as well for most massless integrals; this is plausible assuming that factorization limits relate this massive information to the massless one ${ }^{1}$. Finally, we suggest a geometric interpretation of the IBP vectors (section IVE) which makes generalizations to multiple loops natural.

The article is organized as follows. We start with a heuristic formulation of the central research question in section II] In section III we present important technical background and methods. This includes general coordinate transformations of the loop momenta as well as the discussion of tensor insertions and the unitarity cuts. The off-shell construction of twoloop surface terms is presented in section IV. The reader interested in the final result should be able to read starting from sections IVC.IVE which give the IBP vectors, the formula for surface terms and the vectors' geometric interpretation. In section IVF we speculate about the Lie-algebra structure of the IBP vectors. In section $\mathrm{V}$ we present the on-shell construction of surface terms and count master integrands which serves as valuable cross checks of the off-shell approach. In section IV A and section $\mathrm{VA}$ one-loop examples are given

\footnotetext{
${ }^{1}$ Exceptions may appear when only a single massless leg is attached to a loop.
} 


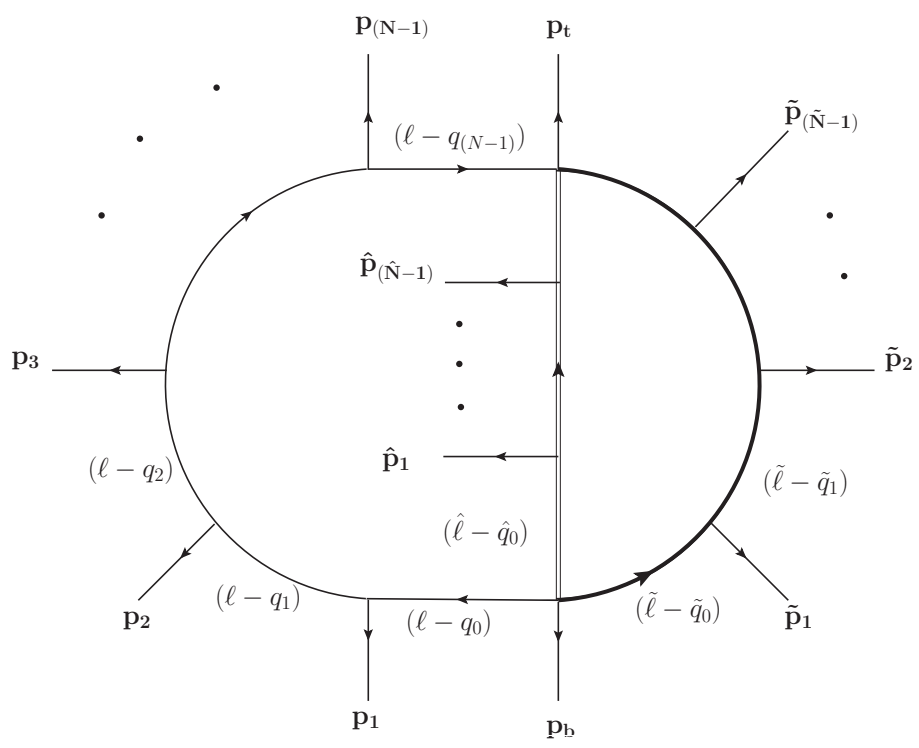

FIG. 1: A generic two-loop integral topology is displayed with the naming conventions used in the main text. In order to reuse structures well known at one-loop level we interpret the two-loop topology as three rungs. The rungs carry loop momentum $\ell, \hat{\ell}$ and $\tilde{\ell}$, and have external momenta $p_{i}, \hat{p}_{i}$ and $\tilde{p}_{i}$ exiting. The rungs are joined in two four-point vertices on the top and bottom with external momenta $p_{t}$ and $p_{b}$ leaving, respectively. Most of our considerations will be focused on the planar case with no external momenta $\hat{p}_{i}$ attached to the central rung at all. Many considerations work analogously for the three rungs and we will often refer to the joint variables by dropping 'hat' and 'tilde' super scripts.

in order to illustrate the off-shell and on-shell constructions, respectively. Finally, we close with a summary and a discussion of a number of interesting future directions. Technical aspects of differential calculus are discussed in an appendix.

\section{SETUP AND NOTATION}

We start with an heuristic introduction of the key structures the we will be dealing with. 


\section{A. Loop integrand decomposition}

We consider two-loop computations with the integral topologies as shown in figure fig. 1. Integrals typically include tensor insertions which are denoted by $t(\ell, \tilde{\ell})$ giving,

$$
\begin{aligned}
\mathcal{I}[t]= & \int d^{D} \ell d^{D} \tilde{\ell} \frac{t(\ell, \tilde{\ell})}{\rho^{0} \cdots \rho^{N-1} \hat{\rho}^{0} \cdots \hat{\rho}^{(\hat{N}-1)} \tilde{\rho}^{0} \cdots \tilde{\rho}^{(\tilde{N}-1)}}, \\
& \text { with } \quad \rho^{i}=\left(\ell-q_{i}\right)^{2}-m_{i}^{2}, \quad \hat{\rho}^{i}=\left(\hat{\ell}-\hat{q}_{i}\right)^{2}-\widehat{m}_{i}^{2}, \quad \tilde{\rho}^{i}=\left(\tilde{\ell}-\tilde{q}_{i}\right)^{2}-\tilde{m}_{i}^{2} .
\end{aligned}
$$

Momentum conservation is imposed $\hat{\ell}=-\left(\ell+\tilde{\ell}-q_{0}-\hat{q}_{0}-\tilde{q}_{0}+\tilde{p}_{b}\right)$. We will work with dimensional regularization keeping the loop-momentum dimensions as free parameters. The tensor insertions are assumed to be polynomial in the loop momenta as it is the case in Feynman amplitudes.

Given a complete basis of numerator tensors $\left\{\tilde{m}_{i}(\ell, \tilde{\ell})\right\}$ with the index $i$ labeling the basis elements, one can evaluate tensor integrals by first decomposing the tensor numerator into the basis,

$$
t(\ell, \tilde{\ell})=\sum_{i \in \text { numerator basis }} \tilde{d}^{i} \tilde{m}_{i}(\ell, \tilde{\ell}),
$$

with loop-momentum independent coefficients $\tilde{d}^{i}$. In a second step one has to integrate all the basis tensor insertions. To this end, typically tensor reduction techniques [23 26, 29] are used to decompose the basis of tensor integrals into a small set of independent master integrals.

Here we aim to shortcut the step of the tensor reduction by constructing a particular numerator basis. Following the strategy of one-loop computations [21, 22], we decompose the numerator tensors into the tensor insertions associated with master integrals $m_{i}(\ell, \tilde{\ell})$ and surface terms $\widehat{m}_{j}(\ell, \tilde{\ell})$, which integrate to zero,

$$
t(\ell, \tilde{\ell})=\sum_{i \in \text { master integrals }} d^{i} m_{i}(\ell, \tilde{\ell})+\sum_{j \in \text { surface terms }} \hat{d}^{j} \widehat{m}_{j}(\ell, \tilde{\ell}),
$$

with the properties,

$$
\mathcal{I}_{i}:=\int d^{D} \ell d^{D} \tilde{\ell} \frac{m_{i}(\ell, \tilde{\ell})}{\rho^{0} \cdots \tilde{\rho}^{(\tilde{N}-1)}}, \quad \int d^{D} \ell d^{D} \tilde{\ell} \frac{\widehat{m}_{j}(\ell, \tilde{\ell})}{\rho^{0} \cdots \tilde{\rho}^{(\tilde{N}-1)}}=0 .
$$

Thus, we directly obtain the decomposition of the initial tensor integral in terms of master integrals $\mathcal{I}_{i}$,

$$
\mathcal{I}[t]=\sum_{i \in \text { master integrals }} d^{i} \mathcal{I}_{i}
$$


while the coefficients $\hat{d}_{j}$ drop out of the final result. The surface terms contribute only prior to the loop integration expressing for example angular correlations.

Within the (numerical) unitarity approaches one works at the integrand level and parametrizations (II.2) of the loop integrands are required. Parametrizations have been developed in the recent years [42-44], however, a decomposition in terms of surface terms and master integrals (II.3) would be important in order to avoid the explicit tensor reduction. The construction of the surface terms has so far not been developed sufficiently and we will provide this missing piece here.

It is important to know the surface terms off-shell, that is all over momentum space and in particular away from the regions of on-shell propagators. This is required on the one hand, to ensure that they in fact integrate to zero in the full loop integrals. On the other hand, even in the unitarity approach off-shell information is required to avoid double counting. That is, given a result for a unitarity cut it has to be subtracted in cuts with fewer on-shell conditions imposed. (E.g. at one-loop results from triple cuts have to be subtracted from two-particle cuts.) This can only be done if the initial cut results can be taken off-shell in a consistent way. A priori a cut, i.e. a product of on-shell tree amplitudes, cannot be taken off-shell. However, once we have used the cut to compute coefficients of an appropriate loop integrand parametrization (using on-shell momenta), we can take the latter off shell and subtract it from daughter cuts. Steps of this kind are explicit or implicit in almost all variants of the unitarity approach, but may possibly be circumvented by introducing phase space integrals or exploiting discrete symmetries [20]. Thus we require an off-shell representation of the loop integrands ideally in terms of surface terms. As a final remark we add that the surface terms have to be algebraic expressions in the loop momenta times propagators. This is the case since they should represent Feynman amplitudes, which have this property.

Integral relations such as IBP identities have all the properties needed for surface terms and can be used when available. This fact is very important for a numerical unitarity approach at higher-loop order. In principle IBP relations may be obtained through standard techniques. However, for the numerical unitarity cuts it is beneficial not to consider an integral basis with doubled propagators. Thus we will construct surface terms from the specialized IBP relations first introduced in ref. [49] which initially do not include integral topologies with doubled propagators. 
An automated construction of the specialized IBP relations has been given in the original article [49] and has been advanced in ref. [50]. Here we prefer to follow an analytic approach, since we require additional control over the expressions. That is we do not only need a compact representation of the surface terms, but we will also need sufficient numerical control when solving for the integral coefficients in all regions of phase space. Nevertheless, it would be instructive to compare the approaches in detail in the future. A geometric onshell construction of specialized IBP relations has been put forward in refs. [52, 53] which is, however, not suitable for our purpose, since we require the full off-shell information of the surface terms. Nevertheless, we will use a related on-shell approach for cross checks below in section $\mathrm{V}$.

\section{B. Adapted coordinates}

Important structures of the loop integrals can be made manifest by using appropriate integration variables. The aim is to change from the loop-momentum components $\left\{\ell^{\mu}, \tilde{\ell}^{\mu}\right\}$ to using the inverse propagators $\left\{\rho^{i}, \tilde{\rho}^{j}, \hat{\rho}^{k}\right\}$ as integration variables [63]. Given the missmatch in the number of propagators and loop-momentum components additional internal variables have to be introduced, which we denote by indexed $\alpha$ 's in the following. The loop integrals are then given by integrations over the inverse propagators in addition to an internal integration over the $\alpha$-coordinates,

$$
\mathcal{I}[t]=\int \frac{[d \rho]}{\rho^{0} \cdots \tilde{\rho}^{(\tilde{N}-1)}} \times t(\rho, \alpha) \mu(\rho, \alpha)[d \alpha],
$$

where $\mu(\rho, \alpha)$ is the non-trivial integration measure from the coordinate change. The expression $[d \rho]$ and $[d \alpha]$ denote the differentials of the integration variables, $\rho$ 's and $\alpha$ 's respectively. The insertion $t(\rho, \alpha)$ is the tensor $t(\ell, \tilde{\ell})$ evaluated in the new coordinates.

It is instructive to consider first the integration over an internal space with the inverse propagators held fixed. From this perspective we can now use the properties of the internal space to organize the computation. To give an example, at one-loop level the internal integration is performed over spheres ${ }^{2}$. We can think of the function $t(\rho, \alpha)$ being decomposed into a linear combination of spherical harmonics. Only the constant function gives a

\footnotetext{
${ }^{2}$ Typically these spheres are part of the complex internal spaces which are tangent bundles of the real spheres $T S_{d}$.
} 
non-vanishing integral, while the higher harmonics integrate to zero. The later can be interpreted as surface terms. Thus surface terms are identified by relating the numerator tensors to spherical harmonics. Fittingly the IBP vectors that generate surface terms turn out to be generators of rotations along the internal space directions. When acting on an insertion $t(\rho, \alpha)$, generators of rotations annihilate the invariant scalar parts, and give nontrivial representation of the rotation group otherwise, that is, non-trivial spherical harmonics. A similar picture holds at higher-loop level but it is also useful to consider the integral in a more formal manner. In formal terms we may relate the task of finding non-trivial integrals to understanding the cohomology of the internal spaces, so that exact forms (total derivatives) in the internal space are related to surface terms, while closed but non-exact forms are related to the non-vanishing master integrals. We will find this perspective useful when considering generalized cuts of the loop integrals.

Not all vanishing integrals arise from surface terms of the internal space alone. For example discrete symmetries can lead to further vanishing integrals. Will not consider the role of discrete symmetries further here, but focus on the identification of surface terms in the earlier sense.

\section{Maximal cuts}

Once we transform to adapted coordinates (II.6) as described above we can naturally make contact with unitarity cuts. Formally, unitarity cuts amount to replacing propagators with delta distributions, $i / \rho \rightarrow \delta(\rho)$. The insertion of the delta distributions localizes the integral to vanishing inverse propagators $\rho^{k}=0$. The Jacobian factors from the coordinate change to adapted coordinates already provides the correct measure for the remaining integrations in the internal variables. (Details about the coordinate change can be found in section III.)

In the maximal cuts of a given integral topology all independent propagators are formally replaced by delta distributions. Once we localize to vanishing inverse propagators, the loop momentum takes on-shell values. Thus the on-shell loop momenta and tensor insertions are obtained by setting all the $\rho^{i}, \tilde{\rho}^{j}$ and $\hat{\rho}^{k}$ to zero. The internal $\alpha$-variables then are the coordinates of the on-shell loop-momentum space. We will refer to this subspace as the maximal-cut phase space in the following. The maximal-cut phase space shares many properties with the surfaces of fixed propagator values allowing to infer properties of the full 
loop integral from on-shell information.

\section{Surface terms as specialized IBP identities}

Surface terms can be obtained from total derivatives starting from (sufficiently regular) vector fields $\left\{u^{\mu}, \tilde{u}^{\nu}\right\}$,

$$
\int d^{D} \ell d^{D} \tilde{\ell}\left[\partial_{\mu}\left(\frac{u^{\mu} t(\ell, \tilde{\ell})}{\rho^{0} \cdots \tilde{\rho}^{(\tilde{N}-1)}}\right)+\tilde{\partial}_{\nu}\left(\frac{\tilde{u}^{\nu} t(\ell, \tilde{\ell})}{\rho^{0} \cdots \tilde{\rho}^{(\tilde{N}-1)}}\right)\right]=0 .
$$

The components of the vector fields are polynomial in the loop momenta to obtain relations between Feynman integrals. Typically doubled propagators appear when the derivatives act on them.

Doubled propagators can be avoided, by a very specific choice of vector-field insertions [49] fulfilling the equations,

$$
\left(u^{\mu} \partial_{\mu}+\tilde{u}^{\nu} \tilde{\partial}_{\nu}\right) \rho^{i}=f^{i}(\ell, \tilde{\ell}) \rho^{i},
$$

for all inverse propagators $\rho^{i}$ and similarly for $\tilde{\rho}^{j}$ and $\hat{\rho}^{j}$ with independent functions $f^{\tilde{j}}(\ell, \tilde{\ell})$ and $f^{\hat{j}}(\ell, \tilde{\ell})$. Due to the chain rule, the doubled propagators are canceled for such special IBP vector fields (II.8). The index $i$ on the right-hand side is not summed over. The functions $f^{i}(\ell, \tilde{\ell})$ are again polynomial in the basic momentum contractions. Typically it is difficult to find this kind of vector field, however, we will point out a simplified construction when adapted coordinates are used.

The relation eq. (II.8) has an interesting on-shell interpretation. When specializing to the on-shell phase spaces with $\rho^{i}=\tilde{\rho}^{j}=\hat{\rho}^{k}=0$, we find that the vector fields $\left\{u^{\mu}, \tilde{u}^{\nu}\right\}$ turn into tangent vectors along the maximal-cut phase spaces: since the right-hand side of eq. (II.8) vanishes, the vectors generate translations that keep the propagators fixed to zero and thus point along on-shell phase space,

$$
\left\{u^{\mu}, \tilde{u}^{\nu}\right\} \underset{\text { on-shell }}{\longrightarrow} \text { phase-space tangent vector }
$$

This property allows one to link off-shell surface terms to on-shell ones as will be discussed in section ПIG, An interpretation of IBP vectors in differential geometry was given as well in [51]. 
Although the on-shell perspective is instructive, we eventually need surface terms that are valid off-shell. To this end we can use the adapted coordinates of the loop integration. Interestingly, the construction of specialized IBP vectors can be solved by inspection. In adapted coordinates the defining equations are,

$$
\begin{aligned}
& \left(u^{a} \partial_{a}+u^{k} \partial_{k}\right) \rho^{i}=u^{i}=f^{i} \rho^{i}, \\
& \Longrightarrow \quad u^{i}=f^{i} \rho^{i} .
\end{aligned}
$$

which follows from $\partial_{k} \rho^{i}=\delta_{k}^{i}$. The $i$ labels are not summed over in the above equation. The notation is explained in more detail in section IIIB and we provide only minimal explanations here. We use the shorthand notation that the index $a$ labels the $\alpha$-variables. Similarly the partial derivative $\partial_{k}$ denotes either of $\left\{\partial_{\rho^{i}}, \partial_{\tilde{\rho}^{i}}, \partial_{\hat{\rho}^{i}}\right\}$. Furthermore, we suppress function arguments in the $f$-functions; $f^{i}:=f^{i}(\rho, \alpha)$.

The form of the IBP vectors allows for a natural geometric interpretation: the $u^{a}$ components generate to horizontal transformations (with fixed $\rho$ ) in the transverse directions and, the $f^{i}$-components induce local conformal transformations in the individual propagator directions.

To summarize, the specialized vector fields have the restriction that the $\rho^{i}$-components are proportional to $\rho^{i}$ and analogously for tilde/hat-coordinates. The general form reads,

$$
\left\{u^{a}, u^{i}\right\}=\left\{u^{a}, \rho^{i} f^{i}\right\} .
$$

(The doubled labels $i$ are not summed over here.) The component functions $\left\{u^{a}, f^{i}\right\}$ are unconstrained apart from the requirement that they are algebraic in the loop momenta (see also section [IIE). As we will see below, given the parametrization of the loop momenta adjusted to the integral topology, it is straight forward to write down the vector fields and consequently the surface terms.

We will often consider vector fields with $f^{i}=0$ and refer to them as horizontal IBP vectors. These vectors induce translations along surfaces of fixed propagator values, which justifies their name. Horizontal vectors are a natural off-shell continuation of the tangent space of the unitarity-cut phase spaces,

$$
\text { phase-space tangent vector } \underset{\text { off-shell }}{\longrightarrow}\left\{u^{a}, 0\right\},
$$

linking on-shell and off-shell data. The conversion between the two is guided by their Liealgebra structure (see sections IV C and IV B). 


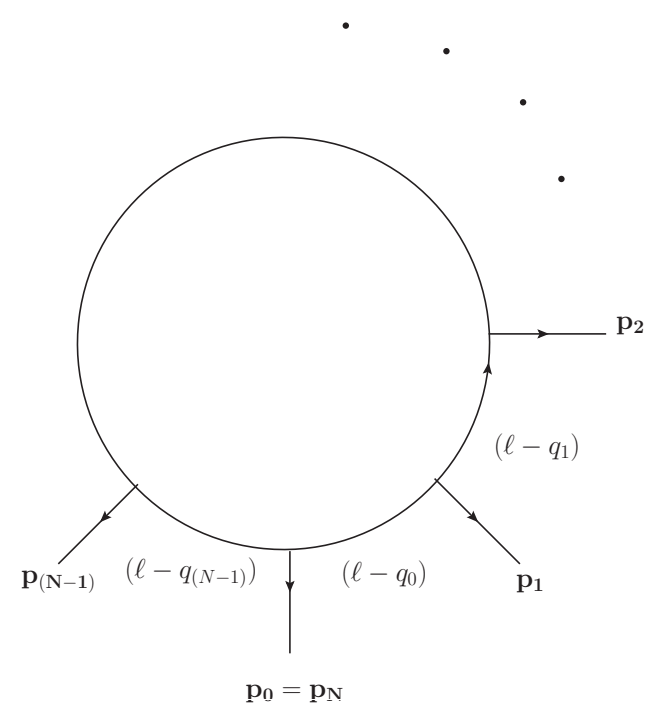

FIG. 2: Conventions for coordinate change for one-loop diagram. Propagator masses and external momenta enter as parameters. The loop momentum $\ell$ is parametrized by the inverse propagators and additional internal variables, in case further parameters are required.

\section{LOOP MOMENTUM PARAMETRIZATIONS}

We will need the explicit form of the general coordinate changes to coordinates adapted to the integral topologies. We will relate the two-loop topology to nested one-loop topologies and reuse one-loop parametrizations. The below considerations hold without restrictions to the dimensionality of the loop momentum which we often suppress. For simplicity we later focus on planar integral topologies and assume generic propagator masses and external masses in order to deal with generic structures rather than special cases.

\section{A. One-loop topologies}

We introduce a particular parametrization of the loop momentum adapted to the topology of the loop diagram in fig. 2, The aim is to change coordinates from the components of the loop momentum $\ell^{\mu}$ to inverse propagators $\rho^{i}$. The construction of such a coordinate transformation can be obtained from ref. [22] which we review below. We use an all-outgoing convention for the external momenta $\left\{p_{i=1, N}\right\}$. The case of generic non-vanishing external and internal masses $\left(m_{i}\right)$ is considered. Using dimensional regularization in $D$ physical dimensions it suffices to consider $N$-gon topologies with $N \leq D+1$. (This allows loop 
momentum dimension to exceed the physical one.) Higher polygons are reducible using Gram-determinant identities [54, 55] and we do not considered them explicitly.

Inverse propagators will be denoted by $\rho^{i}$ and are expressed in terms of the loop momentum $\ell^{\mu}$ by,

$$
\rho^{i}=\left(\ell-q_{i}\right)^{2}-m_{i}^{2}=\left(\ell-\sum_{j=1}^{i} p_{j}\right)^{2}-m_{i}^{2} .
$$

We set the arbitrary constant vector $q_{0}$ to zero for simplicity. When fewer propagators than loop momentum components are present an additional set of internal (angular) coordinates is required which we denote by $\alpha^{i}$. The final result will be the following $D+1$ degrees of freedom,

$$
\begin{array}{cc}
\text { inverse propagators: } & \rho^{0}, \ldots, \rho^{D_{p}}, \\
\text { transverse coordinates: } & \alpha^{1}, \alpha^{2}, \ldots, \alpha^{D_{t}},
\end{array}
$$

with $D_{p}=N-1$ and $D_{p}+D_{t}=D$ and one additional quadratic constraint. The form of the quadratic equation will be discussed below and is given in eq. (III.4).

Explicitly, the coordinate change to adapted coordinates is given by,

$$
\begin{aligned}
& \ell=V(\rho)+ \sum_{a=1}^{D_{t}} n_{a} \alpha^{a}, \quad V(\rho):=\sum_{i=1}^{D_{p}} r_{i} v^{i}, \\
& r_{i}:=-\frac{1}{2}\left(\left(\rho^{i}+m_{i}^{2}-q_{i}^{2}\right)-\left(\rho^{i-1}+m_{i-1}^{2}-q_{i-1}^{2}\right)\right), \quad q_{i}=\sum_{j=1}^{i} p_{j} .
\end{aligned}
$$

Here the vectors $v^{i}$ and $n_{a}$ are elements of the van Neerven-Vermaseren (NV) basis [54] which we introduce momentarily. This basis is adapted to the integral topology and splits momentum space into a $D_{p}=(N-1)$ dimensional 'physical' space spanned by the external momenta and a $D_{t}=\left(D-D_{p}\right)$ dimensional 'transverse' space. For the considered $N$ gons we have $D_{p}$ linear independent external momenta in the set $\left\{p_{i=1, . .,\left(D_{p}+1\right)}\right\}$ due to momentum conservation. (We will also use the convenient notation $p_{0}=p_{N}$.) Using the inverse of the $D_{p}$-dimensional Gram matrix $G_{i j}=\left(p_{i}, p_{j}\right)$ the vectors dual to the external momenta are introduced with $v^{i}=\left(G^{-1}\right)^{i j} p_{j}$ so that they fulfill $\left(v^{i}, p_{j}\right)=\delta_{j}^{i}$. In transverse space an orthonormal basis is used $\left\{n_{a=1, \ldots, D_{t}}\right\}$, with $\left(n_{a}, p_{j}\right)=0$ and $\left(n_{a}, n_{b}\right)=\delta_{a b}$. The transverse basis is not unique and may be changed by (complex) orthogonal rotations. The linear dependence of the vectors $\left\{p_{i}\right\}$ and $\left\{v^{j}\right\}$ implies as well $\left(v^{i}, n_{a}\right)=0$. Explicit analytic 
expressions for the NV basis may also be found in ref. [22]. Particularly compact expressions for the basis decomposition can be obtained in spinor-helicity notation and is inherent in most literature considering analytic unitarity methods (see e.g. [9, 20]).

The parametrization (III.2) solves the linear equations,

$$
\left(p_{i}, \ell\right)=-\frac{1}{2}\left(\left(\rho^{i}+m_{i}^{2}-q_{i}^{2}\right)-\left(\rho^{i-1}+m_{i-1}^{2}-q_{i-1}^{2}\right)\right)=r_{i}
$$

due to the vectors $p_{i}$ and $v^{j}$ being dual. An additional quadratic constraint equation for the internal variables $\alpha^{a}$ is imposed to make sure that the square of the loop momentum gives the inverse propagator,

$$
\begin{gathered}
c(\rho, \alpha)=\left(\ell^{2}-m_{0}^{2}\right)-\rho^{0}=\sum_{a=1}^{D_{t}}\left(\alpha^{a}\right)^{2}+C(\rho)=0, \\
C(\rho)=V(\rho)^{2}-\rho^{0}-m_{0}^{2},
\end{gathered}
$$

with $V(\rho)^{2}=\left(G^{-1}\right)^{i j} r_{i} r_{j}$. With the linear and quadratic equations fulfilled this parametrization returns the correct values for all inverse propagators. Most of the physical information is condensed neatly into the quadratic equation through the Gram matrix as well as its momentum and mass dependence.

A few remarks can be added here: The equation $c(\rho, \alpha)=0$ allows one to eliminate one $\alpha^{a}$ in terms of the inverse propagators and the remaining transverse coordinates. Explicit solutions can be obtained using for example light cone coordinates,

$$
\alpha^{1}=\frac{1}{2}\left(t-\frac{C(\rho)+\sum_{a=3}^{D_{t}}\left(\alpha^{a}\right)^{2}}{t}\right) \quad \alpha^{2}=\frac{i}{2}\left(t+\frac{C(\rho)+\sum_{a=3}^{D_{t}}\left(\alpha^{a}\right)^{2}}{t}\right),
$$

for $D_{t}>1$, and the sum-term is dropped for $D_{t}=2$. Here both $\alpha^{1}$ and $\alpha^{2}$ were traded for a new complex coordinate $t$. For $D_{t}=1$ one can solve for $\alpha^{1}$ directly to obtain,

$$
\alpha^{1}= \pm i \sqrt{C(\rho)}
$$

where the internal manifold degenerates to two distinct points, i.e. a zero-dimensional sphere. However, it is often useful to consider the loop momentum as a hyper surface in $\left\{\alpha^{a}, \rho^{i}\right\}$ space, without using an explicit solution of the quadratic equation inserted.

The inverse coordinate change is given by,

$$
\rho^{i}(\ell)=\left(\ell-q_{i}\right)^{2}-m_{i}^{2}, \quad \alpha^{a}(\ell)=\left(n^{a}, \ell\right)
$$

For the above loop-momentum parametrization the maximal-cut on-shell conditions are implemented by setting the inverse propagators to zero, $\rho^{i} \rightarrow 0$. 


\section{B. Two-loop topologies}

Loop-momentum parametrizations can be obtained by decomposing multi-loop diagrams into sub diagrams which admit one-loop parametrizations. To be specific, any rung in a multi-loop diagram admits a one-loop coordinate transformation yielding sets of internal coordinates and quadratic equations. When the rungs are joined in vertices the momentumconservation conditions impose additional linear equations adding sets of linear equations. Planar as well as non-planar multi-loop parametrizations may be obtained in this way. We will focus first on the planar diagrams.

The generic two-loop topology is displayed in fig. 1, The planar integrals are obtained by specializing to the case where no external momenta are attached to the central rung, i.e. $\hat{N}=0$. In an approach best adapted to planar diagrams we consider the left and right one-loop sub diagrams in the figure and ignore the central rung at first. For the left loop the following external momenta and propagators are used,

$$
\begin{aligned}
& \left\{p_{1}, \ldots, p_{N-1}, p_{N}=-\left(p_{1}+\cdots+p_{(N-1)}\right)\right\} \\
& \rho^{i}=\left(\ell-q_{i}\right)^{2}-m_{i}^{2}, \quad i=1, \ldots N
\end{aligned}
$$

The quantities for the one-loop coordinate transformation $r_{i}, v^{i}, G_{i j}=\left(p_{i}, p_{j}\right)$ and $n_{a}$ are obtained as in section IIIA. Analogously, for the right loop we apply the one-loop transformation with the following list of external momenta inserted,

$$
\begin{aligned}
& \left\{\tilde{p}_{1}, \ldots, \tilde{p}_{\tilde{N}-1}, \tilde{p}_{N}=-\left(\tilde{p}_{1}+\cdots+\tilde{p}_{(\tilde{N}-1)}\right)\right\} \\
& \tilde{\rho}^{i}=\left(\tilde{\ell}-\tilde{q}_{i}\right)^{2}-\tilde{m}_{i}^{2}, \quad i=1, \ldots \tilde{N} .
\end{aligned}
$$

Now we denote the parameters and functions by $\tilde{r}_{i}, \tilde{v}^{i}, \tilde{G}_{i j}=\left(\tilde{p}_{i}, \tilde{p}_{j}\right)$ and $\tilde{n}_{a}$. It is often convenient to distinguish the vectors and derived quantities by their index only, e.g. $\alpha_{\tilde{a}}=\tilde{\alpha}_{\tilde{a}}$.

With these transformations we have the loop momenta parametrized in terms of $\left\{D_{p}=\right.$ $\left.(N-1), \tilde{D}_{p}=(\tilde{N}-1)\right\}$ inverse propagators $\left\{\rho^{i}, \tilde{\rho}^{j}\right\}$ with $1 \leq i \leq D_{p}$ and $1 \leq j \leq \tilde{D}_{p}$. In addition $D_{t}=\left(D-D_{p}\right)$ and $\tilde{D}_{t}=\left(D-\tilde{D}_{p}\right)$ internal coordinates $\left\{\alpha^{a}\right\}$ and $\left\{\tilde{\alpha}^{\tilde{a}}\right\}$ are introduced, respectively.

Explicitly the loop momenta are given by,

$$
\ell=V(\rho)+\sum_{a=1}^{D_{t}} \alpha^{a} n_{a}, \quad \tilde{\ell}=\tilde{V}(\tilde{\rho})+\sum_{\tilde{a}=1}^{\tilde{D}_{t}} \tilde{\alpha}^{\tilde{a}} \tilde{n}_{\tilde{a}}
$$


with $V(\rho)=\sum_{i=1}^{D_{p}} r^{i} v_{i}$ and $\tilde{V}(\tilde{\rho})=\sum_{i=1}^{\tilde{D}_{p}} \tilde{r}^{i} \tilde{v}_{i}$. The internal coordinates have to fulfill the conditions,

$$
c(\rho, \alpha)=\sum_{a=1}^{D_{t}}\left(\alpha^{a}\right)^{2}+C(\rho)=0, \quad \tilde{c}(\tilde{\rho}, \tilde{\alpha})=\sum_{\tilde{a}=1}^{\tilde{D}_{t}}\left(\tilde{\alpha}^{\tilde{a}}\right)^{2}+\tilde{C}(\tilde{\rho})=0,
$$

where $\left.C(\rho)=\left(V(\rho)^{2}-\rho^{0}-m_{0}^{2}\right)\right)$, and $\tilde{C}(\tilde{\rho})=\left(\tilde{V}(\tilde{\rho})^{2}-\tilde{\rho}^{0}-\tilde{m}_{0}^{2}\right)$ setting $q_{0}=\tilde{q}_{0}=0$.

There is one remaining transformation required; in order to express one internal degree of freedom from $\left\{\alpha^{a}, \tilde{\alpha}^{\tilde{a}}\right\}$ in terms of the inverse propagator $\hat{\rho}^{0}$ of the central rung we have the relation,

$$
\begin{aligned}
\hat{c}(\rho, \alpha, \tilde{\rho}, \tilde{\alpha})= & \left(\ell+\tilde{\ell}+p_{b}\right)^{2}-\hat{\rho}^{0}-\widehat{m}_{0}^{2} \\
= & 2\left(\ell+p_{b}, \tilde{\ell}+p_{b}\right)- \\
& -\hat{\rho}^{0}-\widehat{m}_{0}^{2}+\rho^{0}+m_{0}^{2}+\tilde{\rho}^{0}+\tilde{m}_{0}^{2}-p_{b}^{2} .
\end{aligned}
$$

It will be helpful to make the dependence on the $\alpha$-coordinates more explicit, by inserting the form of the loop momenta. We obtain the quadratic equation,

$$
\hat{c}=\alpha^{a} \tilde{\alpha}^{\tilde{a}} \widehat{C}_{a \tilde{a}}+\alpha^{a} \widehat{C}_{a}+\tilde{\alpha}^{\tilde{a}} \widehat{C}_{\tilde{a}}+\widehat{C}
$$

with the definitions,

$$
\begin{aligned}
& \widehat{C}_{a}^{\tilde{k}}=2\left(n_{a}, \tilde{v}^{\tilde{k}}\right), \quad \widehat{C}_{\tilde{a}}^{k}=2\left(v^{k}, \tilde{n}_{\tilde{a}}\right), \quad \widehat{C}_{a \tilde{a}}=2\left(n_{a}, \tilde{n}_{\tilde{a}}\right), \quad \widehat{C}^{k \tilde{k}}=2\left(v^{k}, \tilde{v}^{\tilde{k}}\right), \\
& \widehat{C}_{a}=\widehat{C}_{a \tilde{a}} \tilde{\alpha}_{0}^{\tilde{a}}+\widehat{C}_{a}^{\tilde{k}}\left(\tilde{r}+\tilde{r}_{0}\right)_{\tilde{k}}, \quad \widehat{C}_{\tilde{a}}=\widehat{C}_{\tilde{a} a}^{T} \alpha_{0}^{a}+\widehat{C}_{\tilde{a}}^{k}\left(r+r_{0}\right)_{k}, \\
& \alpha_{0}^{a}=\left(p_{b}, n_{a}\right), \quad \tilde{\alpha}_{0}^{\tilde{a}}=\left(p_{b}, \tilde{n}_{\tilde{a}}\right), \quad r_{0, l}=\left(p_{b}, p_{l}\right), \quad \tilde{r}_{0, \tilde{l}}=\left(p_{b}, \tilde{p}_{\tilde{l}}\right), \\
& \widehat{C}=-\hat{\rho}^{0}-\widehat{m}_{0}^{2}+\rho^{0}+m_{0}^{2}+\tilde{\rho}^{0}+\tilde{m}_{0}^{2}+p_{b}^{2}+\left(r_{k} \tilde{r}_{\tilde{k}}+r_{0, k} \tilde{r}_{\tilde{k}}+r_{k} \tilde{r}_{0, \tilde{k}}\right) \widehat{C}^{k \tilde{k}},
\end{aligned}
$$

where $\widehat{C}_{a}, \widehat{C}_{\tilde{a}}$ and $\widehat{C}$ depend on external kinematics and inverse propagators, while the twoindex terms $\widehat{C}_{a \tilde{a}}, \widehat{C}_{a}^{\tilde{k}}, \widehat{C}_{\tilde{a}}^{k}$ and $\widehat{C}^{k \tilde{k}}$ depend only on the external momenta. The latter matrices quantify the alignment of the physical and transverse spaces of the respective one-loop sub diagrams.

In general, complex orthogonal transformations may be used to rotate the basis vectors of the internal spaces (acting on $a$-labels) and transform the above constraint (III.11) to canonical form. We will discuss the relation of integral topologies and the form of these equations in more detail below. 
For some topologies it is possible to align the basis for the transverse spaces of left and right loop in fig. 1. This leads to a block diagonal form of $C_{a \tilde{a}}$ and vanishing entries in $C_{k \tilde{k}}$ and $C_{a \tilde{k}}$. Similarly the $(D>4)$-dimensional components of the transverse space can be aligned. Rotation symmetries in these independent parts of transverse space are then manifest and simplify the quadratic equations.

In summary, we have traded the loop momenta $\ell^{\mu}$ and $\tilde{\ell}^{\nu}$ for the following coordinates and conditions,

$$
\begin{aligned}
\text { inverse propagators: } & \rho^{0}, \ldots, \rho^{D_{p}}, \tilde{\rho}^{0}, \ldots, \rho^{\tilde{D}_{p}} \text { and } \hat{\rho}^{0}, \\
\text { transverse coordinates: } & \alpha^{1}, \ldots, \alpha^{D_{t}}, \tilde{\alpha}^{1}, \ldots, \tilde{\alpha}^{\tilde{D}_{t}}, \\
\text { quadratic equations: } & c=0, \quad \tilde{c}=0 \quad \text { and } \quad \hat{c}=0 .
\end{aligned}
$$

The quadratic equations $c=\tilde{c}=\hat{c}=0$ have to be solved for the internal coordinates $\alpha$. Instead of finding explicit solutions it is often useful to think of the loop-momentum space as the sub manifolds defined by the quadratic equations in the unconstrained coordinate space of the $\rho$ 's and $\alpha$ 's.

\section{Non-planar parametrization}

Two equivalent ways to consider non-planar topologies will be discussed. The first emphasizes the general structure of multi-loop parametrizations, the second is most convenient for two-loop topologies being an adaptation of the planar setup.

1. Generic parametrization: The non-planar two-loop topology can be viewed as multiple rungs which are joined in vertices; for our notation we refer to fig. 1 (see also later in fig. 3). The individual rungs carry the loop momenta $\ell, \tilde{\ell}$ and $\hat{\ell}$, respectively, which are related by momentum conservation. Each rung can be parametrized using one-loop parametrizations to give three sets of $\alpha$-coordinates and $\rho$-coordinates constrained by three quadratic equations. Compared to the planar case we obtain as well,

$$
\hat{c}(\hat{\rho}, \hat{\alpha})=\sum_{a=1}^{\hat{D}_{t}}\left(\hat{\alpha}^{a}\right)^{2}+\hat{C}(\hat{\rho})=0,
$$

with all functions being natural generalizations of the ones above (III.7) with 'tildes' replaced by 'hats'. In a second step momentum conservation,

$$
\left(\ell-q_{0}\right)+\left(\tilde{\ell}-\tilde{q}_{0}\right)+\left(\hat{\ell}-\hat{q}_{0}\right)+p_{b}=0,
$$


is imposed to relate the transverse coordinates of the individual rungs. In this way one obtains additional coordinates and equations, while the concepts remain the same. Considering multi-loop topologies amounts to adding further rungs and vertices in a similar way.

2. Planar induced parametrization: alternatively we can start from a planar parametrization of the loop momenta and include additional relations to transform the transverse coordinates ( $\alpha$-coordinates) to inverse propagators.

As far as loop momentum parametrizations are concerned rungs can be exchanged, so we can always consider the central rung to have the least amount of external momenta attached; $\hat{N} \leq N$ and $\hat{N} \leq \tilde{N}$. Given that we can have at most eight propagators we have $\hat{N} \leq 2$. Thus, compared to the planar case only one additional inverse propagator variable is required. The constrains from the central rung are explicitly given by,

$$
\begin{aligned}
\hat{c}(\ell, \tilde{\ell}) & =\left(\hat{\ell}-\hat{q}_{0}\right)^{2}-\widehat{m}_{0}^{2}-\hat{\rho}^{0}=\left(\ell+\tilde{\ell}-q_{0}-\tilde{q}_{0}+p_{b}\right)^{2}-\left(\hat{\rho}^{0}+\widehat{m}_{0}^{2}\right), \\
\hat{c}^{\prime \prime}(\ell, \tilde{\ell}) & =\left(\hat{\ell}-\hat{q}_{1}\right)^{2}-\widehat{m}_{1}^{2}-\hat{\rho}^{1}=\left(\ell+\tilde{\ell}-q_{0}-\tilde{q}_{0}+p_{b}+\hat{p}_{1}\right)^{2}-\left(\hat{\rho}^{1}+\widehat{m}_{1}^{2}\right) \\
& =\hat{c}(\ell, \tilde{\ell})+\left(\rho^{0}+\tilde{m}_{0}^{2}\right)-\left(\rho^{1}+\widehat{m}_{1}^{2}\right)+2\left(\ell+\tilde{\ell}-q_{0}-\tilde{q}_{0}+p_{b}, \hat{p}_{1}\right) .
\end{aligned}
$$

While the first constraint is the one already present in the planar topologies, the second one gives one additional linear equation for the loop momenta. It is useful to introduce the simplified constraint explicitly,

$$
\hat{c}^{\prime}(\ell, \tilde{\ell})=\left(\rho^{0}+\tilde{m}_{0}^{2}\right)-\left(\rho^{1}+\widehat{m}_{1}^{2}\right)+2\left(\ell+\tilde{\ell}-q_{0}-\tilde{q}_{0}+p_{b}, \hat{p}_{1}\right) .
$$

We will return to the non-planar cases when discussing explicit IBP vectors in sections IVB and IV C.

\section{A useful integral classification}

It will be useful to refer to individual integral topologies. In principle it is sufficient to specify the number of rung momenta $(N-1, \tilde{N}-1, \hat{N}-1)$, with conventions as in fig. 1 and stating which of the vectors $p_{t}$ and $p_{b}$ vanish. We use the following terminology for the 
topologies,

$$
\begin{array}{rll}
\text { generic: } & p_{t} \neq 0, \quad p_{b} \neq 0 \\
\text { semi-generic: } & p_{t}=0, \quad p_{b} \neq 0, \quad \text { or } \quad p_{t} \neq 0, \quad p_{b}=0 \\
\text { simple: } & p_{t}=0, \quad p_{b}=0 .
\end{array}
$$

These sub classes differ in the number of dependent external momenta which are present in the set $\left\{p_{i}, \tilde{p}_{i}, \hat{p}_{i}\right\}$.

For planar topologies we label the integral topologies by only two numbers which specify external legs $(n, \tilde{n})=(N-1, \tilde{N}-1)$.

If the number of linear independent external momenta is smaller than the physical dimension the transverse spaces of left and right loops overlap and a common transverse space can be defined. We will assume that the transverse NV-vectors are aligned whenever possible.

\section{E. Algebraic Data}

Tensor insertions from Feynman rules give algebraic functions that can be obtained by contracting loop momenta with themselves or with tensors derived from external momenta. These terms are natural in canonical coordinates in momentum space. When using the adapted coordinates we have to make sure that we deal with expressions that arise from coordinate transformations of such algebraic functions.

It turns out that tensor insertions are in fact in one-to-one correspondence with polynomial expressions in the adapted coordinates. This can be shown as follows: On the one hand, adapted coordinates are conventional loop momentum contractions being inverse propagators $\rho^{i}$ or contractions of the form $\alpha^{a}=\left(n_{a}, \ell\right)$. Consequently polynomials in these coordinates are polynomial in the loop momenta. On the other hand, these functions are sufficient to represent all loop momentum contractions: given an expression $\left(t_{\mu_{1} \ldots \nu_{k}} \ell^{\mu_{1}} \ldots \tilde{\ell}^{\nu_{k}}\right)$ we can insert the completeness relations $\left(\delta_{\mu}^{\nu}=n_{\mu}^{a} n^{a \nu}+v_{\mu}^{k} p_{k}^{\nu}\right)$ and $\left(\delta_{\mu}^{\nu}=\tilde{n}_{\mu}^{\tilde{a}} \tilde{n}^{\tilde{a} \nu}+\tilde{v}_{\mu}^{k} \tilde{p}_{k}^{\nu}\right)$ into the contractions with $\ell^{\mu}$ and $\tilde{\ell}^{\nu}$, respectively. The resulting terms $\left(p_{i}, \ell\right)$ and $\left(n_{a}, \ell\right)$ (and similar for the tilde-coordinates) give $r^{i}$ (III.3) and $\alpha^{a}$ respectively. Both are polynomial in $\rho$ 's and $\alpha$ 's, so that any tensor can be expressed in terms of these coordinates.

Thus we can trade any tensor in canonical coordinates for polynomials written in terms 
of inverse propagators and contractions with transverse vectors,

$$
\left(t_{\mu_{1} \ldots \nu_{k}} \ell^{\mu_{1}} \ldots \tilde{\ell}^{\nu_{k}}\right) \longleftrightarrow \prod_{a, \tilde{a}, l, \tilde{l}}\left(\alpha^{a}\right)^{k_{a}}\left(\tilde{\alpha}^{\tilde{a}}\right)^{k_{\tilde{a}}} \times\left(\rho^{l}\right)^{k_{l}}\left(\tilde{\rho}^{\tilde{l}}\right)^{k_{\tilde{l}}}
$$

One may as well include $\hat{\rho}$ and $\hat{\alpha}$ variables, which however can be converted to the above monomials. The variables $k_{i}$ denote non-negative integers.

In order to obtain algebraic surface terms through IBP identities one has to consider algebraic vector fields in momentum space. Such vectors $\left\{u^{\mu}, \tilde{u}^{\nu}\right\}$ are defined to yield algebraic functions $t^{\prime}(\ell, \tilde{\ell})$ upon taking directional derivatives of algebraic functions $t(\ell, \tilde{\ell})$,

$$
\left(u^{\mu} \partial_{\mu}+\tilde{u}^{\nu} \tilde{\partial}_{\nu}\right) t(\ell, \tilde{\ell})=t^{\prime}(\ell, \tilde{\ell})
$$

We may use general coordinate transformations to obtain vector fields in adapted coordinates, however, it is preferable to construct them directly using the above definition in adapted coordinates,

$$
\left(u^{a} \partial_{a}+u^{k} \partial_{k}\right) t(\alpha, \rho)=t^{\prime}(\alpha, \rho)
$$

(We use the shorthand notation for partial derivatives suppressing 'hats' and 'tildes' as in eq. (II.11).) One can show that the components $u^{a}$ and $u^{k}$ of algebraic vector fields have to be algebraic functions by acting one-by-one on the $\alpha$ and $\rho$ coordinates. There is a further condition: above we worked in the coordinate space prior to imposing the conditions $c=\tilde{c}=\hat{c}=0$. Consistent vectors have to be tangent vectors to this surface, which defines the physical momentum space. This means we have to impose the three equations,

$$
\left(u^{a} \partial_{a}+u^{k} \partial_{k}\right)\{c, \tilde{c}, \hat{c}\}=0
$$

to obtain an algebraic vector field. In the non-planar case we have to include one further analogous relation for $\hat{c}^{\prime}$ (II.15).

From these definitions it is clear that multiplying an algebraic vector field by an algebraic function yields again an algebraic vector field.

Finally, given that we deal with integration, we will use differential forms and outer derivatives. As usually, these are defined as linear functions that return the components of vector fields. The differentials,

$$
\left\{d \alpha^{a}, d \rho^{k}\right\}
$$


are algebraic, yielding algebraic functions when acting as linear forms on algebraic vector fields with $d \alpha^{a}\left(\partial_{b}\right)=\delta_{b}^{a}, d \rho^{k}\left(\partial_{l}\right)=\delta_{l}^{k}$ etc. We use again a single label which runs as well over hat and tilde variables.

The 1-forms are not independent due to the relations,

$$
d c=\frac{\partial c}{\partial \alpha^{a}} d \alpha^{a}+\frac{\partial c}{\partial \rho^{k}} d \rho^{k}=0
$$

and analogously for $\tilde{c}$ and the $\hat{c}$ relations. Wedge products can be used to generate the full set of differential forms in adapted coordinates.

\section{F. Function ring and numerator tensors}

We will require a complete set of tensor insertions for a given integral topology limited only by power counting (of typical field theories). Systematic constructions of such a basis of tensor insertions can be found at one-loop level in in ref. [22] (see also [56]) and for multi-

loop topologies in refs. [42 44]. We will review the construction and adjust the notation to our setup. The use of the adapted coordinates makes the construction of irreducible tensor insertions very direct, so that it can often be obtained by hand.

For a given integral topology not all tensor insertions are viewed as independent; inserting an inverse propagator allows one to cancel a propagator and leads to a reduced topology. Thus we can consider numerator tensors modulo inverse propagators. This implies that for the construction of independent numerators inverse propagators are best treated as equivalent to zero $\rho^{k} \sim 0$. Comparing to (III.16) we can proceed in two steps. First, we identify an over complete list of numerator tensors as the polynomials in the $\alpha$ coordinates

$$
\prod_{a, \tilde{a}}\left(\alpha^{a}\right)^{k_{a}}\left(\tilde{\alpha}^{\tilde{a}}\right)^{k_{\tilde{a}}}=\prod_{a, \tilde{a}}\left(n_{a}, \ell\right)^{k_{a}}\left(\tilde{n}_{\tilde{a}}, \tilde{\ell}\right)^{k_{\tilde{a}}},
$$

which are written as well in tensor notation for convenience. The integers $k_{a}$ and $k_{\tilde{a}}$ take positive values limited above by power counting. In a second step we use the relations $(c=\tilde{c}=\hat{c}=0)$, which allow to turn certain polynomials into inverse propagators or monomials of lower degree, thus reducing the independent tensors insertion further.

It is important to note that these equivalence relations amount to imposing the on-shell conditions, with all inverse propagators set to zero. Thus, as far as the construction of a basis of numerator tensors is concerned, linear independent numerator tensors remain independent functions when considered on-shell on the maximal-cut phase spaces. 
However, not all questions can be answered modulo lower topologies and off-shell information is important. For example, considering the tensor insertion of an inverse propagator, we have an 'uninteresting' tensor insertion,

$$
t(\ell, \tilde{\ell})=\rho^{k} \sim 0
$$

and even obtain zero on the maximal cut. However, considering the loop integral, we clearly obtain a scalar integral of lower topology yielding a non-vanishing result,

$$
\int d^{D} \ell d^{D} \tilde{\ell} \frac{\rho^{k}}{\rho^{0} \cdots \tilde{\rho}^{(\tilde{N}-1)}}=\int d^{D} \ell d^{D} \tilde{\ell} \frac{1}{\rho^{1} \cdots \widehat{\rho}^{k} \cdots \tilde{\rho}^{(\tilde{N}-1)}} \neq 0,
$$

with $\widehat{\rho^{k}}$ denoting the omission of the inverse propagator in the numerator. Thus when surface terms are analyzed, we have to work off-shell, although we may obtain guidance from related on-shell questions.

\section{G. Total derivatives and master-integral count}

Here we discuss the relation between computing the total derivatives and cutting all propagators of an integrand. Cutting propagators amounts to replacing the propagators with delta-distributions $(i / \rho \rightarrow \delta(\rho))$. When a tensor integral is written in adapted coordinates (as done in eq. (II.6) ) the operation of cutting omits all propagators as well as the integration measure $[d \rho]$ and sets the inverse propagators to zero,

$$
\int \frac{[d \rho]}{\rho^{0} \cdots \tilde{\rho}^{(\tilde{N}-1)}} \times t(\rho, \alpha) \mu(\rho, \alpha)[d \alpha] \quad \stackrel{\text { cut }}{\longrightarrow} \quad \int t(0, \alpha) \mu(0, \alpha)[d \alpha] .
$$

The function $\mu(\rho, \alpha)$ is a measure factor arising from transforming canonical coordinates $\ell^{\mu}$ to the adapted coordinates $\{\alpha, \rho\}$ and $t(\rho, \alpha)$ denotes the tensor insertion. Terms with some of the cut propagators missing are omitted in the cutting prescription. (One might extend such terms with the necessary inverse propagators and see it vanish when the $\rho$ 's are set to zero.)

For the IBP vectors that do not double propagators we now show that taking total derivatives commutes with the cut operation. That is, propagators drop out of one class of 
terms in the total derivative, which leads to vanishing terms when cuts are applied,

$$
\begin{aligned}
& \int\left[\partial_{i}\left(\frac{u^{i} \mu t}{\rho^{0} \cdots \tilde{\rho}^{(\tilde{N}-1)}}\right)+\partial_{b}\left(\frac{u^{b} \mu t}{\rho^{0} \cdots \tilde{\rho}^{(\tilde{N}-1)}}\right)\right][d \rho, d \alpha] \\
& =\int\left[\frac{\partial_{i}\left(f^{i} \mu t\right)}{\rho^{0} \cdots \widehat{\rho}^{i} \cdots \tilde{\rho}^{(\tilde{N}-1)}}+\partial_{b}\left(\frac{u^{b} \mu t}{\rho^{0} \cdots \tilde{\rho}^{(\tilde{N}-1)}}\right)\right][d \rho, d \alpha] \\
& \stackrel{\text { cut }}{\longrightarrow} \int \partial_{b}\left(u^{b} \mu t\right)[d \alpha] .
\end{aligned}
$$

Here we use the relation $u^{i}=\rho^{i} f^{i}$ and we suppress the arguments for better readability. (In the above equation the labels run as well over hat and tilde values.)

Had we first cut and then used (the pull back of) the IBP vector to obtain a total derivative we obtained the same answer. The special form of the IBP vector fields makes this identification possible in the first place: since they are tangent vectors of the maximalcut phase spaces they are well defined intrinsically on the phase spaces and a pull back is well defined.

It is important to mention that the above reasoning did not involve the $\alpha$-integration itself and is valid as well at the level of integrands (or volume forms considering their Liederivatives [57]).

In general the cut of a vanishing integral does not need to give a surface term on phase space, but might vanish, e.g. due to the choice of the physical integration contour.

\section{On/Off-shell map}

Relating on-shell and off-shell surface terms we can exploit intrinsic properties of the on-shell phase spaces. Every off-shell total derivative from special IBP vectors gives one on the maximal cut (III.25). In formal terms, we obtain exact holomorphic forms of maximal degree for each surface term. We have already seen (section IIIF) that the basis of tensor insertions gives linear independent functions on the maximal-cut spaces. By multiplying with the proper volume element we obtain a holomorphic forms of maximal degree. Given that the coefficient functions are holomorphic and the forms are of maximal degree they are closed; their outer derivative vanishes. Thus surface terms and tensors are given as intrinsic objects of the phase spaces.

Master integrands can be counted on-shell. The number of master integrands is given by the number of independent tensor insertions modulo the number of surface terms. On-shell 
this amounts to the number of closed modulo exact holomorphic forms, that is a topological (global) property of the phase spaces. Thus a topological property, in fact the number of half-maximal cycles, counts master integrands. We will return to counting forms in section $\mathrm{V}$.

\section{CONSTRUCTION OF OFF-SHELL SURFACE TERMS}

We now turn to the main result: the construction of off-shell surface terms which are a central ingredient for the numerical unitarity approach. The construction differs from the one at one-loop level [21, 22, 56] which relied on tensor algebra and symmetries of oneloop integrals. The present construction reproduces the one-loop results and applies also to multi-loop topologies. Put differently, we obtain a complete set of IBP relations which might be valuable beyond its use for the unitarity approach.

The presentation focuses here on planar two-loop topologies, but gives as well non-planar surface terms. Higher-loop generalizations should work in a similar way as we will indicate briefly (section IVE). We consider the four-dimensional construction which yields surface terms that involve the four-dimensional part of the loop momentum. These terms are as well surface terms in $D$ dimensions. Furthermore at one-loop level the four-dimensional numerators were recycled for the $D$-dimensional approach and we believe the same construction works here. Nevertheless, additional IBP vectors can be found beyond the four dimensional ones (see eq. (IV.11)).

The central objects are specialized IBP vectors, which upon computing divergences, are used to obtain the complete set of off-shell surface terms (see section IVD). The master integrals are obtained as convenient tensor insertions in the complement of the surface terms. The construction works topology by topology and reuses one-loop results in sub topologies. In order to introduce the key steps we start with a one-loop example in section IVA. Next, we turn to the two-loop problem. A generating set of IBP vectors is obtained first in adapted coordinates in section IVB and, finally, in canonical notation in section IVC. The latter is the main result of this article and has a very natural geometric interpretation as we discuss in section IVE.

The set of planar generating IBP vectors have the additional property that they leave the integration measure invariant, i.e. their divergence vanishes. This simplifies the computation

of surface terms to taking directional derivatives of irreducible numerator basis. In this way 
an over complete set of surface terms can be obtained which are equivalent to a complete set of IBP relations excluding doubled propagators. We verify the completeness using on-shell techniques in section $\mathrm{V}$,

\section{A. A one-loop example}

The ingredients we need are an irreducible basis of tensor insertions, IBP vectors and the integration measure in order to compute total derivatives. It will turn out, that all IBP vectors can be generated by a set of primitive ones, which we have to consider in detail. Furthermore, it turns out that the primitive IBP vectors leave the volume element and all propagators invariant. Under these circumstances the surface terms are directly obtained by acting with IBP vectors on the irreducible tensor basis. Although these statements hold more generally, we will discuss these steps for the triangle integrals.

\section{Numerator tensors for triangles}

The four degrees of freedom of the loop momentum are parametrized in adapted coordinates by three inverse propagators $\rho^{1,2,3}$ and two internal coordinates $\alpha^{1,2}$ which are constrained by a single quadratic equation (III.4),

$$
c(\alpha, \rho)=\alpha^{1} \alpha^{1}+\alpha^{2} \alpha^{2}+C(\rho)=0,
$$

with $C(\rho)$ given by scalar terms and inverse propagators. The coordinates are related to the loop momenta through the contractions $\alpha^{a}=\left(n^{a}, \ell\right)$ and the definitions of the inverse propagators.

Irreducible numerators are given by polynomials in the $\alpha$-variables (see section 【IF). For standard power-counting we should consider at most cubic powers of the loop momentum

in triangle functions. Thus the tensor insertions $\left(\alpha^{1}\right)^{k_{1}}\left(\alpha^{2}\right)^{k_{2}}=\left(n^{1}, \ell\right)^{k_{1}}\left(n^{2}, \ell\right)^{k_{2}}$ with $\left(k_{1}+\right.$ $\left.k_{2}\right) \leq 3$ suffice. Out of the ten monomials, only seven are linearly independent modulo inverse propagators, as can be seen by using the quadratic equation $c(\alpha, \rho)=0$, which relates inverse propagators and internal coordinates. The three dependent monomials are $\sum_{a}\left(\alpha^{a}\right)^{2} \sim-C(\rho)$ and $\alpha^{1,2} \sum_{a}\left(\alpha^{a}\right)^{2} \sim-\alpha^{1,2} C(\rho)$.

It is convenient to make a linear coordinate change to the coordinates $\alpha^{ \pm}=\left(\alpha^{1} \pm i \alpha^{2}\right)$ 
(and $\left.n^{ \pm}=\left(n^{1} \pm i n^{2}\right)\right)$, so that the constraint equation is given by,

$$
\alpha^{+} \alpha^{-}+C(\rho)=0
$$

The reduction to a minimal numerator basis starting from monomials $\left(\alpha^{+}\right)^{k_{+}}\left(\alpha^{-}\right)^{k_{-}}$then simplifies; it amounts to dropping mixed monomials in $\alpha^{+}$and $\alpha^{-}$, since these are reducible $\alpha^{+} \alpha^{-} \sim-C(\rho)$. The irreducible numerator basis is then given by the seven terms $\left\{1,\left(\alpha^{+}\right)^{m},\left(\alpha^{-}\right)^{l}\right\}$ with $l, k \leq 3$ and thus the integrands,

$$
\frac{\tilde{m}_{ \pm k}(\ell)}{\rho^{1} \rho^{2} \rho^{3}}=\frac{\left(\alpha^{ \pm}\right)^{k}}{\rho^{1} \rho^{2} \rho^{3}}=\frac{\left(n^{ \pm}, \ell\right)^{k}}{\rho^{1} \rho^{2} \rho^{3}} .
$$

We will keep using the light-cone coordinates $\alpha^{ \pm}$below.

\section{Surface terms for triangles}

The main ingredient for this construction are algebraic vector fields obeying the condition (II.10). We start with the ansatz,

$$
u=u^{ \pm} \partial_{\alpha^{ \pm}}+\sum_{i=1,2,3} f^{i} \rho^{i} \partial_{\rho^{i}}
$$

As a simplification we set the function $f^{i}$ to zero and focus on such horizontal vectors, which keep propagators fixed. It turns out that this is no restriction to obtaining a complete set of surface terms here. Imposing the consistency condition (III.19) gives,

$$
\begin{aligned}
& 0=u\left(\alpha^{+} \alpha^{-}+C(\rho)\right)=u^{+} \alpha^{-}+u^{-} \alpha^{+}=0, \\
& \Rightarrow u=i\left(\alpha^{+} \partial_{\alpha^{+}}-\alpha^{-} \partial_{\alpha^{-}}\right)
\end{aligned}
$$

where we gave the simplest solution for the IBP vector. The generic one is obtained by multiplying $u$ with arbitrary polynomials in $\alpha$ and $\rho$ variables.

It is instructive to rewrite the obtained IBP vector in canonical momentum variables,

$$
\begin{gathered}
u=i\left(\alpha^{+} \partial_{\alpha^{+}}-\alpha^{-} \partial_{\alpha^{-}}\right)=\left(\alpha^{1} \partial_{\alpha^{2}}-\alpha^{2} \partial_{\alpha^{1}}\right) \\
=\left[\left(n^{1}, \ell\right)\left(n^{2}\right)^{\mu}-\left(n^{2}, \ell\right)\left(n^{1}\right)^{\mu}\right] \partial_{\mu} .
\end{gathered}
$$

A number of remarks can be added here: 1) The IBP vectors are generators of rotations in the transverse space spanned by the $n^{a}$ vectors of the NV basis. 2) By construction the 
vector $u$ leaves the inverse propagators invariant. In canonical coordinates this follows from the anti symmetry of the vector and the property $\left(n^{a}, p_{i}\right)=0$,

$$
\begin{aligned}
u\left(\ell^{2}\right) & =2\left[\left(n^{1}, \ell\right)\left(n^{2}, \ell\right)-\left(n^{2}, \ell\right)\left(n^{1}, \ell\right)\right]=0, \\
u\left(\left(\ell-q_{i}\right)^{2}\right) & =u\left(\ell^{2}-2\left(\ell, q_{i}\right)\right)=0-2\left[\left(n^{1}, \ell\right)\left(n^{2}, q_{i}\right)-\left(n^{2}, \ell\right)\left(n^{1}, q_{i}\right)\right]=0 .
\end{aligned}
$$

3) The divergence of the IBP vector vanishes. In canonical coordinates it is straight forward to compute the divergence of a vector field, since the volume element is constant ${ }^{3}$ and one can verify,

$$
\partial_{\mu} u^{\mu}=0
$$

This property simplifies the computation of the divergences of generic IBP vectors which are obtained by multiplying the primitive ones by algebraic tensors $t(\ell)$,

$$
\partial_{\mu}\left(t(\ell) u^{\mu}\right)=u^{\mu} \partial_{\mu} t(\ell)
$$

Thus the numerators of the triangle surface terms are directional derivatives of generic tensors $t(\ell)$ with respect to the primitive IBP vector. Given that the $\rho$-components have been set to zero the propagator terms do not have to be considered when computing the total derivative (or divergence).

After these remarks it is straight forward to put together the IBP relations of surface terms. First of all, we write down a redundant set of IBP vectors by multiplying the generator $u$ with the irreducible numerator basis, $\left(\alpha^{ \pm}\right)^{m} u^{\mu}$. Then we calculate the divergences and obtain,

$$
\frac{\widehat{m}_{ \pm k}(\ell)}{\rho^{1} \rho^{2} \rho^{3}}=\partial_{\mu}\left[\frac{\left(\alpha^{ \pm}\right)^{k} u^{\mu}}{\rho^{1} \rho^{2} \rho^{3}}\right]=\frac{u^{\mu} \partial_{\mu}\left(\alpha^{ \pm}\right)^{k}}{\rho^{1} \rho^{2} \rho^{3}}=\frac{ \pm k\left(\alpha^{ \pm}\right)^{k}}{\rho^{1} \rho^{2} \rho^{3}}= \pm k \frac{\left(n^{ \pm}, \ell\right)^{k}}{\rho^{1} \rho^{2} \rho^{3}} .
$$

The only irreducible numerator that does not appear is the constant one $(k=0)$, which is associated with the master integral. This is the well known result for triangle integrals. Often surface terms are represented as symmetric traceless tensors; the tensors $\left(n_{\mu}^{ \pm} \cdots n_{\nu}^{ \pm}\right)$ are symmetric as well as traceless since the vectors $n^{ \pm}$have vanishing norm.

\footnotetext{
${ }^{3}$ In general coordinate systems the divergence of a vector field is obtained as its Lie-derivative acting on the volume element [57].
} 


\section{B. Special two-loop IBP vectors - adapted coordinates}

For a given topology we transform to the adapted coordinates and use the observation (II.11) that the IBP vectors have to take the form (II.10) with their $\rho^{i}$-components being proportional to the associated inverse propagators $\rho^{i}$. With this ansatz we impose the conditions (III.19) for algebraic vector fields. Given the simplicity of the equations, we can solve them by inspection.

\section{One-loop IBP vectors}

We require first vectors that leave the quadratic equations $c=\tilde{c}=0$ of the individual loops invariant. Interesting IBP vectors are:

(a) Generators of rotations,

$$
u_{[a b]}=\alpha_{a} \partial_{b}-\alpha_{b} \partial_{a}
$$

and analogously for the tilde-coordinates.

(b) Vectors with non-trivial $\rho$-components,

$$
u_{i}=\alpha^{a} \rho^{i} \partial_{\rho^{i}}-\rho^{i}\left(\partial_{\rho^{i}} c\right) \partial_{a},
$$

(no summation over the index $i$ ). We do not need this class of vectors here. Nevertheless, it would be interesting to understand the role of these vectors better, as they may be used to relate tensor insertions of distinct integral topologies.

(c) D-dimensional vectors,

$$
u_{a}=\alpha^{a} \mu^{b} \partial_{\mu^{b}}-\left(\mu^{b} \mu^{b}\right) \partial_{\alpha^{a}}
$$

where the labels $b$ are summed over. Some explanations are required here. Going beyond four dimensions the dimensionality of the loop momentum is increased leading to additional transverse directions. The additional transverse coordinates are conventionally called $\mu^{b}:=\alpha^{b+D_{t}}$. Rotation-invariance in the $(D-4)$ dimensional directions is maintained and made manifest for the above vectors. We give these vectors for completeness but will not consider them further here.

For our purposes only the vectors of type (a) will be important. We will refer to such vectors, which do not contain components in the propagator directions as horizontal vectors. 


\section{Two-loop IBP vectors}

Next, we consider the central rung and form linear combinations of the rotation generators (IV.9) which leave the rung equation $\hat{c}=0$ invariant:

(a) For more than two transverse variables in a given loop we find that the rung-condition singles out a rotation axis ${ }^{4}$ and we can write the linear combination,

$$
u_{[a b c]}=e_{[a \mid} u_{\mid b c]}
$$

where the notion $[a b c]$ denotes the labels' anti-symmetric combinations. The rotation axis is obtained by acting with the generator on the rung relation (III.10),

$$
e_{a}=\partial_{a} \hat{c}
$$

The anti-symmetric index structure makes sure that the rung condition is annihilated by the vector ( $(\underline{I V .12})$.

(b) Diagonal rotations on the $\alpha$ space and $\tilde{\alpha}$ spaces give valid IBP vectors, whenever the internal spaces are lined up in at least two directions and the rung relation is quadratic in the respective $\alpha$-variables. This is the case in some semi-generic or simple topologies (see section VB 2). (The coordinates in quadratic parts of the rung relations must not appear in linear terms.) The vectors are then,

$$
u_{[a b]}^{\operatorname{diag}}=u_{[a b]}+\tilde{u}_{[a b]} .
$$

(c) Crossed rotations: these can appear, whenever both sides give rise to at least two internal $\alpha$-coordinates each,

$$
u_{[a b][c d]}=\tilde{e}_{c d} u_{[a b]}-e_{a b} \tilde{u}_{[c d]} .
$$

Here the anti-symmetric quantities $e_{a b}$ and $\tilde{e}_{a b}$ are given by the infinitesimal transformations of the rung equation,

$$
\tilde{e}_{a b}:=\tilde{u}_{[a b]} \hat{c}, \quad \text { and } \quad e_{a b}=u_{[a b]} \hat{c}
$$

\footnotetext{
${ }^{4}$ Rotations are enumerated by the inequivalent $(D-2)$-dimensional planes they leave invariant. We use the term axis in an intuitive way to state that the set of planes is restricted to the ones that contain the specified axis vector.
} 
(d) Additional vectors can be constructed including inverse-propagator derivatives as well as expressions including $D$-dimensional components. We will not discuss these vectors here.

Multiplying the above vectors with generic tensors gives further valid IBP vectors,

$$
\prod_{a, \tilde{a}} \alpha_{a}^{k_{a}} \tilde{\alpha}_{\tilde{a}}^{\tilde{k}_{\tilde{a}}} \times u
$$

which can be used to construct an over-complete list of surface terms. We will discuss the construction of surface terms in more detail in section IVD.

We refer to the vectors (a)-(c) as horizontal, given that they generate motions that do not alter the propagator values. These vectors turn into tangent vectors of the unitaritycut phase spaces, when on-shell conditions are imposed. At the same time they may be viewed as an off-shell continuation of the tangent bundles of the on-shell phase spaces to generic propagator values. The continuation is not unique. Here the IBP equation (II.8) with a vanishing right hand side as well as the underlying Lie-algebra structure allow one to control the off-shell continuation.

\section{Non-planar IBP vectors}

The number of transverse variables limits the IBP vectors that can be introduced. The relevant topologies are given by the $(N, \tilde{N}, \hat{N})=(2,2,2)$ and $(N, \tilde{N}, \hat{N})=(2,2,3)$. All remaining non-planar topologies have no unconstrained internal degrees of freedom and no surface terms with transverse coordinates only may be obtained. The $(2,2,2)$-topology has six internal coordinates $\left(\alpha^{1,2,3}\right.$ and $\left.\tilde{\alpha}^{1,2,3}\right)$ which together with four constrains give a two dimensional internal space. The $(2,2,3)$-topology start from five internal coordinates $\left(\alpha^{1,2,3}\right.$ and $\tilde{\alpha}^{1,2}$ ) and remain with a one parameter after all constraints are imposed. We base the construction on the parametrizations inherited from the planar case (III.15) where two constraint from the central rung are imposed, $\hat{c}=\hat{c}^{\prime}=0$.

One type of vectors may be introduced for both topologies,

$$
\begin{gathered}
u=e_{[\tilde{a} \tilde{b}]} e_{[c d]}^{\prime} u_{[f g]}-e_{[c d]} e_{[\tilde{a} \tilde{b}]}^{\prime} u_{[f g]}+e_{[c d]} e_{[f g]}^{\prime} u_{[\tilde{a} \tilde{b}]} \\
-e_{[\tilde{a} \tilde{b}]} e_{[f g]}^{\prime} u_{[c d]}+e_{[f g]} e_{[\tilde{a} \tilde{b}]}^{\prime} u_{[c d]}-e_{[f g]} e_{[c d]}^{\prime} u_{[\tilde{a} \tilde{b}]}, \\
e_{[a b]}=u_{[a b]}(\hat{c}), \quad e_{[a b]}^{\prime}=u_{[a b]}\left(\hat{c}^{\prime}\right) .
\end{gathered}
$$


Here we use 'tilde' labels to distinguish data of the left and right loop. For the $(N, \tilde{N}, \hat{N})=$ $(2,2,2)$ topology a second such vector can be constructed by exchanging all variables of left and right loop $a \leftrightarrow \tilde{a}$.

Two additional primitive vectors may be constructed for the (2,2,2)-topology,

$$
\begin{aligned}
u & =\tilde{e} u_{a b c}-e u_{\tilde{a} \tilde{b} \tilde{c}}, \\
u^{\prime} & =\tilde{e} u_{a b c}^{\prime}-e u_{\tilde{a} \tilde{b} \tilde{c}}^{\prime},
\end{aligned}
$$

with the various symbols defined by,

$$
\begin{aligned}
u_{a b c} & =e_{[a \mid} \alpha_{|b|} \partial_{\mid c]}, \quad u_{a b c}^{\prime}=e_{[a \mid}^{\prime} \alpha_{|b|} \partial_{\mid c]}, \\
e_{a} & =\partial_{a}(\hat{c}), \quad e_{a}^{\prime}=\partial_{a}\left(\hat{c}^{\prime}\right), \quad e=e_{[a \mid} \alpha_{|b|} e_{\mid c]}^{\prime}, \quad \tilde{e}=e_{[\tilde{a} \mid} \alpha_{|\tilde{b}|} e_{\mid \tilde{c}]}^{\prime} .
\end{aligned}
$$

and similarly for tilde-coordinates.

\section{Special two-loop IBP vectors - standard notation}

The one-loop rotation generators are given in standard notation by,

$$
u_{[k l]}=\left(\ell, n_{[k \mid}\right)\left(n_{\mid l]}, \partial\right),
$$

which allow to construct the generic two loop vectors by composition. This vector matches the one in eq. (IV.9) up to a coordinate change. This can be verified by comparing their action on the internal coordinates and propagators.

\section{Two-loop IBP vectors}

The above primitive IBP vectors (IV.12)-(玨.15) can be given as well in canonical notation using the loop momenta $\ell, \tilde{\ell}$ and $\hat{\ell}$. The momenta are related by momentum conservation $\hat{\ell}=-\left(\ell-q_{0}+\tilde{\ell}-\tilde{q}_{0}+p_{b}\right)+\hat{q}_{0}$, but to simplify expressions we use the dependent momentum $\hat{\ell}$. In the following we set the arbitrary shift vectors to zero $q_{0}=\tilde{q}_{0}=\hat{q}_{0}=0$ to simplify the expressions.

The two-loop vectors are given by the following three types:

(a) Rotation around an axis,

$$
u_{[i j k]}=\left(\hat{\ell}, n_{[i \mid}\right)\left(\ell, n_{|j|}\right)\left(n_{\mid k]}, \partial\right),
$$


or with tilde-expressions exchanged with non-tilde ones. Hidden in $\left(\hat{\ell}, n_{a}\right)$ we have terms containing the contractions $\left(\tilde{\ell}, n_{a}\right)$ which can be rewritten using the completeness relation $\delta_{\nu}^{\mu}=\sum_{\tilde{a}} \tilde{n}^{\tilde{a} \mu} \tilde{n}_{\nu}^{\tilde{a}}+\sum_{\tilde{i}} \tilde{p}_{\tilde{i}}^{\mu} \tilde{v}_{\nu}^{\tilde{i}}$ to give $\left[\left(\tilde{\ell}, \tilde{n}_{\tilde{a}}\right)\left(\tilde{n}_{\tilde{a}}, n_{a}\right)+\left(\tilde{\ell}, \tilde{p}_{\tilde{i}}\right)\left(\tilde{v}^{\tilde{i}}, n_{a}\right)\right]$. The later terms contain the terms $\left(\tilde{\ell}, \tilde{p}_{\tilde{i}}\right)$ and thus off-shell information when expressed through inverse propagators.

(b) Diagonal rotations,

$$
u_{[k l]}=\left(\ell, n_{[k \mid}\right)\left(n_{\mid l]}, \partial\right)+\left(\tilde{\ell}, \tilde{n}_{[k \mid}\right)\left(\tilde{n}_{\mid l]}, \tilde{\partial}\right)
$$

(c) Crossed rotations,

$$
\begin{aligned}
u_{[i j][k l]}=\left(\tilde{\ell}, \tilde{n}_{[k \mid}\right)\left(\tilde{n}_{\mid l]}, \hat{\ell}\right)\left(\ell, n_{[i \mid}\right)\left(n_{\mid j]}, \partial\right)- \\
\quad-\left(\ell, n_{[i \mid}\right)\left(n_{\mid j]}, \hat{\ell}\right)\left(\tilde{\ell}, \tilde{n}_{[k \mid}\right)\left(\tilde{n}_{\mid l]}, \tilde{\partial}\right) .
\end{aligned}
$$

It follows from the symmetrization properties and the definitions of the transverse space vectors, that all these vectors in fact annihilate inverse propagators. This confirms that the listed vectors are valid specialized IBP vectors.

Multiplying the above vectors with generic tensors gives further IBP vectors,

$$
\prod_{i, j}\left(n_{i}, \ell\right)^{k_{i}}\left(\tilde{n}_{j}, \tilde{\ell}\right)^{k_{j}} \times u
$$

which can be used to give an over-complete list of surface terms. For the planar topologies (section IVC2) we have checked for that this list is sufficient to generate all surface terms (see section $\mathrm{V})$.

\section{Vectors and topologies}

The dimensionality and the alignment of the transverse spaces of a given integral topology determines which of the above vectors are present. We collect this information in table \. We use the notation of section IIID and label integral topologies by the pairs $(n, \tilde{n})=$ $(N-1, \tilde{N}-1)$.

Type- $a$ vectors can be constructed, if the dimension of one-loop transverse spaces at least three; $n<2$ or $\tilde{n}<2$. Type- $b$ vectors can be constructed, if the common transverse space 
is at least two-dimensional. Vectors of type- $c$ can be constructed, if each of the transverse spaces is at least two-dimensional; $n<3$ and $\tilde{n}<3$.

For example, the $(1,2)$-topology has a physical space spanned by $\left\{p_{1}, \tilde{p}_{1}, \tilde{p}_{2}\right\}$. The left transverse space is $D-1=3$ dimensional and the right transverse space $D-2=2$ dimensional. For the 'generic' and 'semi-generic' topologies the external momenta $\left\{p_{1}, \tilde{p}_{1}, \tilde{p}_{2}\right\}$ are linear independent and thus they span three-dimensional space. The common transverse space is then one-dimensional. We can construct type- $a$ vectors, since the left transverse space is three-dimensional. The common transverse space is one-dimensional and no type- $b$ vectors exist. Type- $c$ vectors exist, given that each loop has at least two transverse directions. For the 'simple' $(1,2)$-topology the span $\left\{p_{1}, \tilde{p}_{1}, \tilde{p}_{2}\right\}$ is only two-dimensional and the common transverse space has two dimensions and type- $b$ vectors can be constructed.

\section{Non-planar IBP vectors}

As discussed above the relevant topologies are given by the $(N, \tilde{N}, \hat{N})=(2,2,2)$ and $(N, \tilde{N}, \hat{N})=(2,2,3)$.

The first type of vectors may be introduced for both types of topologies.

$$
\begin{aligned}
u= & \tilde{e}_{[i j]} e_{[k l]}^{\prime} u_{[m n]}-e_{[k l]} \tilde{e}_{[i j]}^{\prime} u_{[m n]}+e_{[k l]} e_{[m n]}^{\prime} \tilde{u}_{[i j]} \\
& -\tilde{e}_{[i j]} e_{[m n]}^{\prime} u_{[k l]}+e_{[m n]} \tilde{e}_{[i j]}^{\prime} u_{[k l]}-e_{[m n]} e_{[k l]}^{\prime} \tilde{u}_{[i j]},
\end{aligned}
$$

with the auxiliary definitions,

$$
\begin{array}{ll}
u_{[i j]}=\left(n_{[i \mid}, \ell\right)\left(n_{\mid j]}, \partial\right), & \tilde{u}_{[i j]}=\left(\tilde{n}_{[i \mid}, \tilde{\ell}\right)\left(\tilde{n}_{\mid j]}, \tilde{\partial}\right), \\
e_{[i j]}=\left(n_{[i \mid}, \ell\right)\left(n_{\mid j]}, \hat{\ell}\right), & e_{[i j]}^{\prime}=\left(n_{[i \mid}, \ell\right)\left(n_{\mid j]}, \hat{p}_{1}\right), \\
\tilde{e}_{[i j]}=\left(\tilde{n}_{[i \mid}, \tilde{\ell}\right)\left(\tilde{n}_{\mid j]}, \hat{\ell}\right), & \tilde{e}_{[i j]}^{\prime}=\left(\tilde{n}_{[i \mid}, \tilde{\ell}\right)\left(\tilde{n}_{\mid j]}, \hat{p}_{1}\right) .
\end{array}
$$

We used the rung momentum $\hat{\ell}$ which may be obtained from $\ell$ and $\tilde{\ell}$ by momentum conservation. The arbitrary shift vectors $q_{0}, \tilde{q}_{0}$ and $\hat{q}_{0}$ are set to zero for simplicity. For the $(N, \tilde{N}, \hat{N})=(2,2,2)$ topology a second such vector can be constructed by exchanging all variables of left and right loop.

Two additional primitive vectors can be constructed for the $(2,2,2)$-topology,

$$
\begin{aligned}
u & =\tilde{e} u_{i j k}-e \tilde{u}_{i j k}, \\
u^{\prime} & =\tilde{e} u_{i j k}^{\prime}-e \tilde{u}_{i j k}^{\prime},
\end{aligned}
$$




\begin{tabular}{||c||c|c|c||}
\hline \multicolumn{1}{||c||}{} & \multicolumn{3}{c||}{ types of IBP vectors } \\
\cline { 2 - 4 }$($ legs left,legs right) & generic & semi-generic & simple \\
\hline \hline$(0,0)$ & $a, b, c$ & - & - \\
\hline$(0,1)$ & $a, b, c$ & $a, b, c$ & - \\
\hline$(0,2)$ & $a, b, c$ & $a, b, c$ & $a, b, c$ \\
\hline$(0,3)$ & $a$ & $a$ & $a$ \\
\hline \hline$(1,1)$ & $a, b, c$ & $a, b, c$ & $a, b, c$ \\
\hline$(1,2)$ & $a, c$ & $a, c$ & $a, b, c$ \\
\hline$(1,3)$ & $a$ & $a$ & $a$ \\
\hline$(2,2)$ & $c$ & $c$ & $c$ \\
\hline \hline$(2,3)$ & - & - & - \\
\hline
\end{tabular}

TABLE I: Here we display how the IBP-vector types are associated with integral topologies. Three types of IBP vectors are presented in section IVC. These are associated with rotations around an axis (type a), diagonal rotations (type b) and crossed rotations (type c). The integral topologies determine which and how many of the three types of vectors can be constructed. Here we summarize this information for planar integral topologies. Only planar topologies of the master integrals with a central rung are considered. The topologies are specified by (legs left,legs right); this refers to the attached legs on the left/right loop (see section IIID). The $(2,3)$ topologies have no unconstrained internal coordinates and, thus, no horizontal IBP vectors can be constructed.

with the various symbols defined by,

$$
\begin{aligned}
& u_{i j k}=\left(n_{[i \mid}, \hat{\ell}\right)\left(n_{|j|}, \ell\right)\left(n_{\mid k]}, \partial\right), \quad u_{i j k}^{\prime}=\left(n_{[i \mid}, \hat{p}_{1}\right)\left(n_{|j|}, \ell\right)\left(n_{\mid k]}, \partial\right) \text {, } \\
& \tilde{u}_{i j k}=\left(\tilde{n}_{[i \mid}, \hat{\ell}\right)\left(\tilde{n}_{|j|}, \tilde{\ell}\right)\left(\tilde{n}_{\mid k]}, \tilde{\partial}\right), \quad \tilde{u}_{i j k}^{\prime}=\left(\tilde{n}_{[i \mid}, \hat{p}_{1}\right)\left(\tilde{n}_{|j|}, \tilde{\ell}\right)\left(\tilde{n}_{\mid k]}, \tilde{\partial}\right), \\
& e=\left(n_{[i \mid}, \hat{\ell}\right)\left(n_{|j|}, \ell\right)\left(n_{\mid k]}, \hat{p}_{1}\right), \quad \tilde{e}=\left(\tilde{n}_{[i \mid}, \hat{\ell}\right)\left(\tilde{n}_{|j|}, \tilde{\ell}\right)\left(\tilde{n}_{\mid k]}, \hat{p}_{1}\right) .
\end{aligned}
$$

\section{Surface from IBP vectors for planar integrals}

The surface terms are obtained by computing the divergence with the above IBP vectors inserted into the loop integrands (IV.25).

A number of properties of the vectors lead to simplified expressions in the planar topolo- 
gies. All vectors annihilate the inverse propagators. Furthermore, the divergence of the above primitive IBP vectors (IV.22,IV.24) vanishes: the total derivative of rotation generators vanishes because of its antisymmetric index structure. Similarly the diagonal rotation generators (IV.23) as well as the ones around an axis (IV.22) have vanishing divergence. Finally the crossed rotation generators (IV.24) give,

$$
\partial_{\mu} u_{[i j][k l]}^{\mu}+\tilde{\partial}_{\nu} \tilde{u}_{[i j][k l]}^{\nu}=\left(\tilde{\ell}, \tilde{n}_{[k \mid}\right)\left(\tilde{n}_{\mid l]}, n_{[j \mid}\right)\left(\ell, n_{\mid i]}\right)-\left(\ell, n_{[i \mid}\right)\left(n_{\mid j]}, \tilde{n}_{[l \mid}\right)\left(\tilde{\ell}, \tilde{n}_{\mid k]}\right)=0 .
$$

In general, inverse propagators have to be included in computing surface terms from IBP vectors (II.7), however, with the IBP vectors annihilating all inverse propagators the derivation simplifies to taking the divergence,

$$
\begin{aligned}
\widehat{m}_{u}= & \partial_{\mu}\left(\prod_{a, \tilde{a}}\left(n_{a}, \ell\right)^{k_{a}}\left(\tilde{n}_{\tilde{a}}, \tilde{\ell}\right)^{k_{\tilde{a}}} \times u^{\mu}\right)+\tilde{\partial}_{\nu}\left(\prod_{a, \tilde{a}}\left(n_{a}, \ell\right)^{k_{a}}\left(\tilde{n}_{\tilde{a}}, \tilde{\ell}\right)^{k_{\tilde{a}}} \times \tilde{u}^{\nu}\right) \\
= & \left(u^{\mu} \partial_{\mu}+\tilde{u}^{\nu} \tilde{\partial}_{\nu}\right)\left(\prod_{a, \tilde{a}}\left(n_{a}, \ell\right)^{k_{a}}\left(\tilde{n}_{\tilde{a}}, \tilde{\ell}\right)^{k_{\tilde{a}}}\right) .
\end{aligned}
$$

This is the main result of this article providing an over complete list of surface terms; these are given by the action of the above IBP vectors (IV.22,IV.24) on the basis tensors (III.21). The master integrals have to be taken from the complement and can be chosen as the lowest

powers of the irreducible numerators. In section $\mathrm{V}]$ we validate that this set of surface terms is in fact complete. For the non-planar case the divergence-containing terms of the primitive vectors are not expected to vanish, but can easily be included.

It is interesting to note here that numerators $\widehat{m}_{u}(\overline{\text { IV.30 }})$ can be inserted as well for topologies with the powers of the propagators altered,

$$
\int d^{D} \ell d^{D} \tilde{\ell} \frac{\widehat{m}_{u}(\ell, \tilde{\ell})}{\left(\rho^{0}\right)^{k_{0}} \cdots\left(\tilde{\rho}^{(\tilde{N}-1)}\right)^{k_{(\tilde{N}-1)}}}=0,
$$

for the horizontal vectors $u$ defined in section IVC and section $\amalg I \mathrm{C}$.

\section{E. Geometric interpretation}

The picture that emerges is that horizontal IBP vectors are particular infinitesimal rotations in the transverse spaces. In fact, we may decompose a generic multi-loop diagram into rungs, which join in vertices as displayed in fig. 3. For each rung we can use adapted 


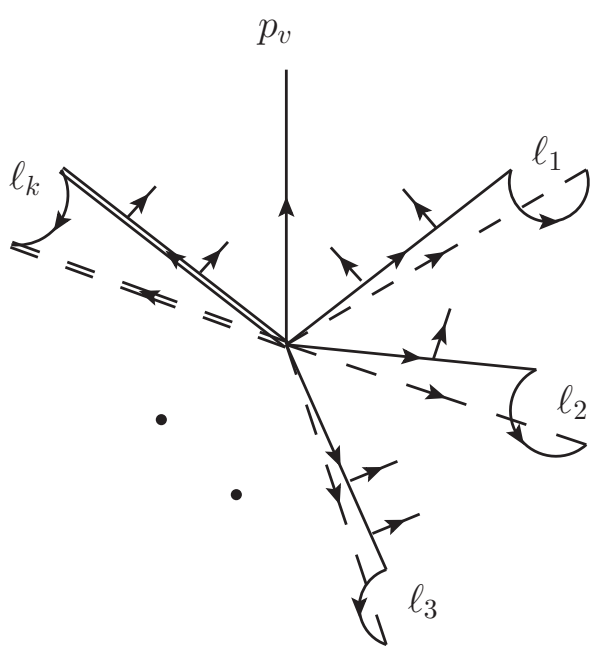

FIG. 3: The junction of internal lines of a generic multi-loop diagram is displayed. The loop momenta $\ell_{i}$ and the external momentum $p_{v}$ join at the vertex. The momenta are constrained by momentum conservation. Each of the loop legs (here referred to as rungs) may have external momenta entering, which is indicated by small attached arrows. IBP vectors generate rotations in the respective transverse spaces of the individual rungs. Vertices impose interesting compatibility conditions between the rotations of the individual rungs. Via momentum conservation individual rotations of the rungs with loop momenta $\left\{\ell_{1}, \ldots, \ell_{k-1}\right\}$ lead to a resulting transformation of $\ell_{k}$. The resulting transformation must be a rotation within this rung's transverse space to give a valid IBP vector.

coordinates, identify an associated transverse space and, as in the one-loop problems, obtain simple quadratic equations (III.4). Candidate IBP vectors have to keep the individual quadratic equation invariant and thus must be generators of rotations. (As above we set propagator components of the vectors to zero.) At the vertices momentum conservation has to be imposed yielding additional linear constraints. To discuss this case further we assume a vertex that joins $k$ rungs with additional external momentum $p_{v}$ entering,

$$
\ell_{k}=-\left(\ell_{1}+\ldots+\ell_{k-1}+p_{v}\right)
$$

Typical IBP vectors have to be combinations of the individual rotation generators, which rotate within the individual transverse spaces. Momentum conservation then imposes an interesting compatibility condition between the rotations; it requires that the individual rotations of the rung momenta $\ell_{i \neq k}$ lead to a resulting rotation in the transverse space of 
the dependent momentum $\ell_{k}$.

There are a number of interesting questions that should be addressed. Maybe the most fundamental being the question if it suffices to consider horizontal IBP vectors with vanishing $\rho$-components. Furthermore, it would be interesting to take further advantage of the representation theory of the generators. For example it should be possible to decompose polarization states with respect to their rotation properties under the IBP transformations in order to reduce state sums in the loops. We leave these and other questions to the future.

\section{F. Lie-algebra structure}

We add a speculative discussion about the fundamental question, in how far the physical amplitudes are determined by unitarity? We want to address this question leaving the master integrals aside and consider their coefficients as the physical quantities.

In order to obtain the integral coefficients, we first try to reconstruct the integrand. Given that perturbative field theories are algebraic and power counting conditions hold, we have a finite number of terms which can be fixed on-shell on generalized cuts. Terms that are missed in one unitarity cut have to be proportional to inverse propagators and can be determined if the set of all generalized cuts is considered. Knowing the integrand, we still have to identify the physical terms which correspond to the coefficients of the master integrals.

Given the loop integrand, the physical information can be extracted once we have a split up into surface terms and master integrands; it is given by the coefficients $d_{i}$ of the master integral. The analogous statement holds for the numerators of unitarity cuts; once the split up into surface terms (closed forms) and the remaining on-shell master integrands is known, we can obtain the physical information of the unitarity cut as the master coefficients $d_{j}^{\text {on-shell }}$.

However, a prior, it is not clear that the same physics is contained in the two kinds of master coefficients, $d_{i}$ and $d_{j}^{\text {on-shell }}$. The core structure for identifying master integrands are the above IBP vectors in their on-shell or off-shell versions. Each one of them determines a set of surface terms and master integrals. Now, the fact that we find a correspondence between the vectors, means that the on-shell and off-shell integrand decompositions can be lined up and with it the physical coefficients. In this way we believe that the off-shell continuation of the IBP vectors is a fundamental structure allowing the unitarity cuts to organise the amplitudes' physics, possibly according to transcendentality. 
We close this discussion with summarizing, that two structures play an important role for the construction of the IBP vectors: the IBP relation (II.8) and the Lie-algebra structure. These do not only allow to construct the vectors and surface terms but also provide a way to lift on-shell to off-shell information. The off-shell continuation works as follows: we first match the on-shell vectors to rotation generators and then extend the generators off-shell maintaining their Lie-algebra structure. The remaining ambiguity proportional to inverse propagators is eliminated by considering additional unitarity cuts and the horizontality condition.

\section{COUNTING MASTER INTEGRANDS ON-SHELL}

Here we use an on-shell approach to validate our main results presented in the previous section. To this end we compare two distinct ways to obtain master integrands for each of the discussed integral topologies. In the first approach we compare the linear span of special IBP relations (surface terms) with the span of irreducible tensor insertions. The difference of the two gives the master integrands which we count,

$$
N_{\text {master }}=N_{\text {irreducible }}-N_{\text {ibp-relation }} \text {. }
$$

We count modulo inverse propagators which is equivalent to counting the functions when pulled back to the maximal-cut phase spaces.

In the second approach we consider the structure of on-shell phase spaces directly to count master integrals. The logic of the on-shell approach goes as follows. We have shown in section III G that all surface terms from IBP relations are turned into on-shell surface terms, that is exact forms on the maximal cuts. Thus the set of all exact forms has to encompass the ones from IBP relations $N_{\text {ibp-relation }} \leq N_{\text {exact }}$. Furthermore, we have discussed in section that independent tensor insertions remain independent functions on the maximal cuts. Thus, when the tensor integrals are (maximally) cut, they yield linear independent holomorphic forms of maximal degree on the maximal-cut phase spaces

$$
N_{\text {irreducible }}=N_{\text {closed }} .
$$

To exploit these observations we construct the complete set of exact forms of maximal degree (a combinatorial problem) and then compare the linear spans of exact and closed 
forms. The difference of the two is expected to give at least a lower bound on the number of master integrals,

$$
N_{\text {master }}^{\prime}=N_{\text {closed }}-N_{\text {exact }}
$$

and is a topological property of the on-shell phase spaces. We will confirm that we obtain the same number of master integrals $N_{\text {master }}=N_{\text {master }}^{\prime}$ in both approaches (section $\mathrm{VC}$ ). Given that the number of irreducible tensors matches in both approaches, we conclude that we have found the maximal set of independent surface terms

$$
N_{\text {exact }}=N_{\text {ibp-relation }}
$$

Thus, we verify that the set of primitive IBP vectors is complete and generates all surface terms. Similarly, the set of surface terms is complete, in the sense that their complement in the irreducible numerator tensors, i.e. the master integrands, are distinguishable by unitarity cuts. Nevertheless, symmetric integration contours can still lead to a small number of linear combinations of master integrands which integrate to zero.

Throughout the construction we assume generic, non vanishing propagator and external masses. This tries to accomplish two things: on the one hand this mimics the $D$-dimensional questions and, on the other hand, the phase spaces of the maximal cuts are then most regular allowing to use standard differential calculus.

We have checked the approach for consistency. We have two distinct software implementations producing identical results. We have anchored the results with the examples we know of: we reproduce the (well known) one-loop counts of master integrals and spurious terms. Furthermore, we reproduce the count of nine master integrals in the double-box refs. [52, 53] and four master integrands [58] in the sunset topology with generic masses. The results are consistent with the integral count presented earlier in ref. [59] which, however, considers the four-point topologies which often have vanishing external masses.

Some of the on-shell methodology has become available in a recent publication [52] for selected multi-loop topologies. These involve typically maximal cuts yielding a onedimensional phase spaces. We work in a different direction considering generic multidimensional phase spaces, exploit it to inspire the off-shell construction and compute the number of master integrals for all planar integral topologies. 


\section{A. A one-loop example}

We first explain the approach in a simple one-loop example namely the triangle functions. The setup is analogous to the off-shell construction in section IV A. There the complete set of surface terms has been obtained as well as the irreducible tensor insertions. We can read off the result,

$$
N_{\text {master }}=N_{\text {irreducible }}-N_{\text {ibp-relation }}=1,
$$

with $N_{\text {irreducible }}=7$ and $N_{\text {ibp-relation }}=6$.

We next turn to the on-shell approach. Upon imposing the on-shell conditions $\rho^{i}=0$, we are left with the on-shell phase space parameterized by $\alpha^{ \pm}$which are constrained by the simplified quadratic equation $c=\alpha^{+} \alpha^{-}+C(0)=0$.

\section{Algebraic function ring and differentials}

The on-shell phase space is one-dimensional and we have to use 1-forms as integration measures. We first construct all 0-forms, i.e. algebraic functions and then take outer derivatives to obtain the exact 1-forms. The ring of functions is generated by $\alpha^{+}$and $\alpha^{-}$,

$$
\left(\alpha^{+}\right)^{k^{+}}\left(\alpha^{-}\right)^{k^{-}}, \quad k^{+}+k^{-} \leq 3
$$

with the rank of the monomials restricted by power counting.

The independent functions are obtained by using the relation $\alpha^{+} \alpha^{-}=-C$ with the shorthand notation $C:=C(0)$. Thus, as in the off-shell case the independent functions are given by $1,\left(\alpha^{+}\right)^{m}$ or $\left(\alpha^{-}\right)^{n}$; whenever a mixed term of $\alpha^{+}$and $\alpha^{-}$appears it can be turned into the constant $C$. The set of all differentials are generated by the freely generated ring of formal expressions $d \alpha^{+}$and $d \alpha^{-}$

$$
\left(\alpha^{+}\right)^{k^{+}}\left(\alpha^{-}\right)^{k^{-}} d \alpha^{ \pm}, \quad k^{+}+k^{-} \leq 3
$$

The linear independent differentials are obtained by imposing the on-shell conditions $d c=0$ and $c d \alpha^{ \pm}=0$ for the differentials. We can first reduce to fourteen terms $\left(\alpha^{ \pm}\right)^{k} d \alpha^{ \pm}$reducing the coefficient functions. Then we use the relation, $\alpha^{+} d \alpha^{-}+\alpha^{-} d \alpha^{+}=0$ to relate the forms $\left(\alpha^{+}\right)^{(k>1)} d \alpha^{-}=C\left(\alpha^{+}\right)^{k-2} d \alpha^{+}\left(\right.$and $\left.\left(\alpha^{-}\right)^{(k>1)} d \alpha^{+}=C\left(\alpha^{-}\right)^{k-2} d \alpha^{-}\right)$to obtain the seven independent forms $\left(N_{\text {closed }}=7\right)$,

$$
\left(\alpha^{+}\right)^{k^{+}} d \alpha^{+}, \quad\left(\alpha^{-}\right)^{k^{-}} d \alpha^{-}, \quad \alpha^{+} d \alpha^{-}=-\alpha^{-} d \alpha^{+}, \quad k^{ \pm} \leq 3 .
$$


The set of all exact differentials is given by taking outer derivatives on the function ring (V.3) with $\left(k^{+}+k^{-}\right) \leq 4$. Losing the constant function we obtain six $\left(N_{\text {exact }}=6\right)$ exact forms

$d\left(\left(\alpha^{ \pm}\right)^{k}\right)=k\left(\alpha^{ \pm}\right)^{k-1} d \alpha^{ \pm}$. These forms are linear independent and no further reduction is needed. Compared to the set of all 1-forms the exact ones do not include $\alpha^{-} d \alpha^{+}$making it a representative of a master integral as the only non-exact form. Thus we obtain,

$$
N_{\text {master }}^{\prime}=N_{\text {closed }}-N_{\text {exact }}=1
$$

with $N_{\text {closed }}=7$ and $N_{\text {exact }}=6$. In more general cases it is often convenient to count the combined sets of closed and exact forms as well as the exact forms, with a relaxed powercounting restriction on the exact forms to avoids boundary effects. We observe $N_{\text {master }}=$ $N_{\text {master }}^{\prime}=1$ which is the well known result. From the topology of the maximal-cut phase space this is expected, since we have one non-trivial cycle to which the form $\alpha^{+} d \alpha^{-}$is dual to.

We close with a remark: we can make contact with the standard on-shell notation: we may solve the quadratic equation $\alpha^{+} \alpha^{-}=-C$ and use the explicit parametrization $\alpha^{+}=t$ and $\alpha^{-}=-C / t$. The set of tensor insertions is given by $t^{k}$ and $(C / t)^{k}$. It is straight forward to write down all closed forms by acting with the outer derivative on the functions $t^{k}$ with $k$ being a positive or negative integer. From the generated forms $\left(t^{k} d t / t\right)$ only $d t / t$ cannot be obtained from acting on the functions $t^{k}$ since $d t / t=d \ln (t)$ with $\ln (t)$ being non-algebraic. Again we obtain a single non-trivial closed form. We conclude that there is one master integrand with the on-shell representation $d t / t$ and six surface terms $t^{k} d t / t$ with $k \neq 0$ and $|k| \leq 3$

\section{B. Two-loop computations}

Computing the off-shell data is straight forward following the instructions of section IVD, For the on-shell construction a number of ingredients are needed. We need to give a classification of the relevant integral topologies and their on-shell equations. Furthermore, we need an algorithm to construct exact and closed forms. We will turn to these points in the following. 


\section{Setup}

The maximal cuts are obtained using the adapted coordinates (section III) by setting the inverse propagators to zero. Consequently, the maximal-cut phase spaces are parameterized by the internal coordinates $\alpha^{a}$ and $\tilde{\alpha}^{\tilde{a}}$ with quadratic equations ([II.8 III.9) of the following form imposed,

$$
\begin{aligned}
& c=\alpha^{a} \alpha^{a}+C=0, \\
& \tilde{c}=\tilde{\alpha} \tilde{\alpha}^{\tilde{a}}+\tilde{C}=0, \\
& \hat{c}=\widehat{C}_{a \tilde{a}} \alpha^{a} \tilde{\alpha}^{\tilde{a}}+\widehat{C}_{\tilde{a}} \tilde{\alpha}^{\tilde{a}}+\alpha^{a} \widehat{C}_{a}+\widehat{C}=0,
\end{aligned}
$$

where $C, \tilde{C}, \widehat{C}, \widehat{C}_{a}, \widehat{C}_{\tilde{a}}$ and the matrices $\widehat{C}_{a \tilde{a}}$ are determined from the explicit kinematic configuration of external momenta and the integral topology. In the non-planar case we had a further equation $\hat{c}^{\prime}=0$ but we focus on the planar cases now.

The function space is generated (non-minimally) by tensor insertions evaluated on the maximal cut, which is given here by the monomials,

$$
\prod_{a, \tilde{a}} \alpha_{a}^{k_{a}} \tilde{\alpha}_{\tilde{a}}^{\tilde{k}_{\tilde{a}}}
$$

The set of independent (irreducible) functions is obtained by imposing the on-shell conditions (V.5) . Algebraically one considers the above monomials modulo multiples of the on-shell conditions; that is modulo the ideal generated by the on-shell conditions. We assume a fixed set of external momenta and masses. The coefficients can be viewed as constants and we can use sufficiently generic integer-valued expressions for simplicity.

Power counting restricts the monomials. Assuming that a vertex contributes at most a single power of loop momentum we obtain the restriction $\sum_{a} k_{a} \leq(N+1), \sum_{\tilde{a}} \tilde{k}_{\tilde{a}} \leq(\tilde{N}+1)$ and $\sum_{a} k_{a}+\sum_{\tilde{a}} \tilde{k}_{\tilde{a}} \leq N+\tilde{N}$. For simplicity we will impose the power-counting constraint in the following, although the approach is not limited to power-counting renormalizable theories.

\section{Equations and topology}

The quadratic equations ( $(\overline{V .5})$ simplify for certain topologies. This in turn changes the symmetry content, which is characterized by the IBP vectors, and consequently the number 
of master integrals. The final form of the quadratic equations will be given below for the individual topologies.

The first two quadratic equations are invariant under orthogonal transformation of the internal space variables $\alpha^{a}$ and $\tilde{\alpha}^{\tilde{a}}$. These rotations may be used to transform the rungequation to a canonical form. Here we use the NV vectors to fix the form of the rung equation and insert integer coefficients for our computations.

We distinguish integral topologies as well as integrals with and without momenta $\left(p_{b}\right.$ and $p_{t}$ in our conventions) attached to the top and bottom rung vertices. These external momenta in the rung equations influence the alignment of the transverse and physical spaces of the respective loops. Three classes of integrals are distinguished, where we restrict ourselves to the diagrams with $n=(N-1)$ legs and $\tilde{n}=(\tilde{N}-1)$ legs attached to left and right loop, respectively. Without loss of generality we assume $(\tilde{n} \geq n)$ configurations. The dimensions of the left and right transverse spaces are $(D-n)$ and $(D-\tilde{n})$ respectively. Apart from the distinct dimensionality we assume that the transverse spaces differ by a generic rotation from one another.

1. Generic case: these correspond to $(n, \tilde{n})$-leg diagrams with non-vanishing $p_{b}$ and $p_{t}$. The dimension of the common physical space is $\operatorname{Min}(n+\tilde{n}, D)$ and the common transverse space has dimension $\operatorname{Max}(D-n-\tilde{n}, 0)$. The quadratic equation for the central rung is given by,

$$
\begin{gathered}
\sum_{i=1}^{D-n-\tilde{n}}(\alpha-\widehat{C})^{i}(\tilde{\alpha}-\widehat{C})^{i}+\sum_{b, \tilde{b}=D-n-\tilde{n}+1}^{D-\tilde{n}} \widehat{C}_{b \tilde{b}} \alpha^{b} \tilde{\alpha}^{\tilde{b}}+ \\
+\sum_{\tilde{b}=D-n-\tilde{n}+1}^{D-\tilde{n}} \widehat{C}_{\tilde{b}} \tilde{\alpha}^{\tilde{b}}+\sum_{b=D-n-\tilde{n}+1}^{D-n} \alpha^{b} \widehat{C}_{b}+\widehat{C}=0
\end{gathered}
$$

In the $(D-n-\tilde{n})$-dimensional common transverse space we can line up the basis vectors $n_{i}$ and $\tilde{n}_{i}$ to obtain a unit diagonal bilinear term; this justifies the form of the first term. We have $\tilde{n}$ remaining transverse directions from the left loop and $n \leq \tilde{n}$ from the right loop. The left transverse basis can then be rotated, so that $(\tilde{n}-n)$ transverse vectors $n_{b>(D-\tilde{n})}$ of the left loop are orthogonal to the $\tilde{n}$ basis vectors $\tilde{n}_{b}$ of the right loop. The $n$ directions $\left\{n_{b}, \tilde{n}_{b}\right\}$ with $(D-n-\tilde{n}+1) \leq b \leq(D-\tilde{n})$ lie at generic angles with respect to each other. The latter gives rise to the generic $n \times n$ matrix $\widehat{C}_{b \tilde{b}} \sim\left(n_{b}, \tilde{n}_{\tilde{b}}\right)$. The linear terms represent the orientation of the external 
momenta with respect to the transverse bases. The vectors $\widehat{C}^{i}$ arise from the inner products $\left(p_{b}, n_{i}\right)=\left(p_{b}, \tilde{n}_{i}\right)$. The constant vectors $\widehat{C}_{b}$ and $\widehat{C}_{\tilde{b}}$ arise from contractions of the transverse space vectors of the left/right-loop with the physical directions.

2. Semi-generic case: by this topology we mean that only one rung vertex has external momentum attached. Without loss of generality we assume $p_{b}=0$.

$$
\sum_{i=1}^{D-n-\tilde{n}} \alpha^{i} \tilde{\alpha}^{i}+\sum_{b, \tilde{b}=D-n-\tilde{n}+1}^{D-\tilde{n}} \widehat{C}_{b \tilde{b}} \alpha^{b} \tilde{\alpha}^{\tilde{b}}+\sum_{\tilde{b}=D-n-\tilde{n}+1}^{D-\tilde{n}} \widehat{C}_{\tilde{b}} \tilde{\alpha}^{\tilde{b}}+\sum_{b=D-n-\tilde{n}+1}^{D-n} \alpha^{b} \widehat{C}_{b}+\widehat{C}=0
$$

The only change compared to the generic case is that the constant vectors $\widehat{C}^{i}$ vanish since $p_{b}=0$.

3. Simplest case: by this topology we mean that $p_{b}=p_{t}=0$. Relative to the generic case this implies the following simplifications. The vectors $\widehat{C}^{i}$ vanish and, furthermore, the external momenta attached to the left and right loop are linearly dependent. Thus the dimension of the common transverse space increases by one,

$$
\sum_{i=1}^{D-n-\tilde{n}+1} \alpha^{i} \tilde{\alpha}^{i}+\sum_{b, \tilde{b}=D-n-\tilde{n}+2}^{D-\tilde{n}} \widehat{C}_{b \tilde{b}} \alpha^{b} \tilde{\alpha}^{\tilde{b}}+\sum_{\tilde{b}=D-n-\tilde{n}+2}^{D-\tilde{n}} \widehat{C}_{\tilde{b}} \tilde{\alpha}^{\tilde{b}}+\sum_{b=D-n-\tilde{n}+2}^{D-n} \alpha^{b} \widehat{C}_{b}+\widehat{C}=0
$$

These equations represent the essence of the two-loop integrands and give a moduli space of the on-shell conditions. Here the equations are required as the input for the computation of the exact forms as well as the irreducible numerators.

\section{The algorithm}

We will now construct holomorphic differential forms of maximal degree on the unitaritycut phase spaces and relate them to surface terms obtained as IBP relations. We will compare and count both results as a consistency check and a validation of the completeness of the surface terms computed earlier.

The considerations go as follows. Tensor insertions appear as volume elements (or forms) on the internal space following the split up (ㅍ.6). Furthermore, although the coordinates $\left(\alpha^{a}, \tilde{\alpha}^{\tilde{a}}\right)$ are complex valued the volume element arises from $t(\ell, \tilde{\ell}) d^{D} \ell d^{D} \tilde{\ell}$, which is holomorphic so that it suffices to consider holomorphic forms and functions. (In practice this 
means that we do not need to use $\bar{\alpha}^{a}, d \bar{\alpha}^{a}$ etc.) Since the holomorphic forms have maximal degree they are in fact closed.

In addition to the closed forms we need to consider exact forms given that all IBP vectors generate exact forms on-shell (section IIIG). Both types of forms are defined on the on-shell spaces which are algebraic varieties. It will be sufficient to consider algebraic forms on these spaces (see also section VB 4).

Eventually we count master integrals. These are given by closed forms that are non-exact, i.e. that are not surface terms.

We will first generate over complete sets of closed and exact forms in a combinatorial way. These sets of forms will be denoted by $\hat{\Omega}_{\text {closed }}$ and $\hat{\Omega}_{\text {exact }}$, respectively. In a second step we will reduce $\hat{\Omega}_{\text {closed }}$ and $\hat{\Omega}_{\text {exact }}$ by vanishing forms $\Omega_{\text {zero }}$ to obtain the linear independent sets $\Omega_{\text {closed }}=\hat{\Omega}_{\text {closed }} / \Omega_{\text {zero }}$ and $\Omega_{\text {exact }}=\hat{\Omega}_{\text {exact }} / \Omega_{\text {zero }}$. The vanishing forms $\Omega_{\text {zero }}$ have nontrivial algebraic expressions but vanish when the on-shell conditions are taken into account, e.g. the one-form $d c$ is equivalent to zero on-shell $d c \sim 0$ (III.20). The form degree matches the dimensionality of the phase space which we denote by $m$. For convenience we label the onshell conditions by an index $i ;\left\{c_{i}\right\}=\{c, \tilde{c}, \hat{c}, \ldots\}$ and enumerate the transverse coordinates by a single label $i$ with $\left\{\alpha^{i}\right\}=\left\{\alpha^{a}, \tilde{\alpha}^{\tilde{a}}\right\}$.

Algebraic $n$-forms can be obtained in a combinatorial way; first of all one has to take outer products of the independent algebraic 1-forms to obtain a basis of $n$-forms. Next one multiplies these forms by monomials in the transverse coordinates to obtain forms up to a fixed polynomial rank. Such $n$-forms are given by,

$$
\omega_{n}=\left(\prod_{i}\left(\alpha^{i}\right)^{k_{i}}\right) d \alpha^{j_{1}} \wedge \cdots \wedge d \alpha^{j_{n}} .
$$

The symbols $k_{i}$ denote non-negative integers. Typically we are interested in the linear span of such forms over the complex numbers.

The vanishing form $\Omega_{z e r o}$ are given as the linear span of the following $m$-forms:

1. Algebraic $m$-forms $\omega_{m}$ multiplied by an on-shell relation $c_{i}$,

$$
\omega_{z e r o}=c_{i} \omega_{m}
$$

2. Forms obtained from outer products with a differential of the on-shell relation,

$$
\omega_{\text {zero }}=d c_{i} \wedge \omega_{m-1}
$$


The exact forms $\omega_{\text {exact }} \in \hat{\Omega}_{\text {exact }}$ are generated by taking outer derivatives on holomorphic forms of one degree less than the volume form,

$$
\omega_{\text {exact }}=d \omega_{m-1}
$$

The closed forms $\hat{\Omega}_{\text {closed }}$ are given by the holomorphic algebraic $m$-forms (V.7).

With closed, exact and vanishing forms available, the independent forms are obtained as equivalence classes of the relations,

$$
\omega_{m} \sim \omega_{m}+\omega_{z e r o}
$$

We thus obtain $\Omega_{\text {closed }}=\hat{\Omega}_{\text {closed }} / \Omega_{\text {zero }}$ and $\Omega_{\text {exact }}=\hat{\Omega}_{\text {exact }} / \Omega_{\text {zero }}$.

Finally, we can count master integrals $\Omega_{\text {master }}$ as closed forms that are non-exact. We compare the linear spans of $\Omega_{\text {closed }}$ and $\Omega_{\text {exact }}$; we quotient $\Omega_{\text {closed }}$ by $\Omega_{\text {exact }}$,

$$
N_{\text {master }}^{\prime}=\operatorname{dim}\left(\Omega_{\text {master }}\right), \quad \Omega_{\text {master }}=\Omega_{\text {closed }} / \Omega_{\text {exact }}
$$

In principle the polynomial order of the forms is restricted by power counting. However, it is often simpler to relax the power counting conditions. At least in the present cases the number of master integrals is not reduced by power counting conditions. Furthermore, it is convenient to compute the dimensionality of $\Omega_{\text {master }}$ indirectly by

$$
N_{\text {master }}^{\prime}=\operatorname{dim}\left(\Omega_{\text {closed }} \cup \Omega_{\text {exact }}\right)-\operatorname{dim}\left(\Omega_{\text {exact }}\right) .
$$

This helps to avoid boundary effects due to limiting the polynomial rank of the forms; for a fixed polynomial degree the set of exact forms does not need to be subset of the closed ones.

We implemented the above computations by translating to a related polynomial problems (see section $\mathrm{A}$ ). In fact we can treat the basis forms as auxiliary coordinates. Exact as well as closed forms then appear as polynomials. The vanishing forms can be interpreted as algebraic relations generating an ideal. The constructions of exact and closed forms then transforms to reducing the polynomials associated with exact and closed forms in the extended affine coordinate ring by the ideal of the vanishing forms.

\section{Discussion of the on-shell measure}

The counting of master integrands in the previous subsections was based on algebraic differential forms on the unitarity-cut phase space. However, while the off-shell volume 
element is algebraic, the on-shell one arises from integrating out delta-function constraints. Thus the cutting procedure leads to holomorphic forms that differ from algebraic ones. For the smooth spaces we are dealing with, the volume element carries a non-singular factor. (This factor can be obtained from the coordinate changes but we will not need it here.)

Nevertheless, we argue that the algebraic count provides sufficient information. The number of master integrals is given by a topological quantity and we assume that the algebraic computation is sufficient to obtain its value.

\section{Master integrand counting}

We have implemented the construction of closed and exact algebraic differential forms in Mathematica [60]. As input we used the on-shell conditions given in section VB2 and we computed the expected number of master integrals $N_{\text {master }}^{\prime}$. The computations were performed for generic numerical coefficient matrices in the quadratic equations.

In a second computation we constructed the primitive IBP vectors using adapted coordinates. We then computed surface terms using these vectors. (Technically we computed Lie-derivatives of the closed algebraic forms with respect to the IBP vectors.) We confirm that the number of master integrals $N_{\text {master }}$ matches in both approaches,

$$
N_{\text {master }}^{\prime}=N_{\text {master }}
$$

for all considered planar topologies. As a reference we give the counts of the master integrands below in table II.

For completeness we give as well the total number of irreducible numerator tensors, which were obtained here by reducing the function ring of the internal coordinates by the quadratic relations section $\mathrm{VB} 2$, These numbers match the results in the literature [44, 61? ? ]. This check is based on the same input equations as used for computing surface terms and counting master integrands. Thus comparing irreducible numerators validates our input equations.

We included as well results for diagrams with bubbles on internal lines. These topologies come with doubled propagators to start with. The corresponding results are marked with an asterisk and referred to as 'simple' $(0, n)$ topologies. In the 'simple' topologies no legs are attached to the vertices of the central rung.

The count of the master integrals is useful by itself but, furthermore, confirms the com- 


\begin{tabular}{||c||c|c|c||c||}
\hline \multirow{2}{*}{\multicolumn{1}{||c||}{$($ legs left,legs right $)$}} & \multicolumn{3}{c||}{ \# master integrands } & \multirow{2}{*}{} \\
\cline { 2 - 5 } & generic & semi-generic & simple & irred. tensors \\
\hline \hline$(0,0)$ & 4 & - & - & 42 \\
\hline$(0,1)$ & 4 & 1 & - & 80 \\
\hline$(0,2)$ & 6 & 1 & $1^{*}$ & 65 \\
\hline$(0,3)$ & 2 & 2 & $2^{*}$ & 18 \\
\hline$(1,1)$ & 9 & 5 & 1 & 111 \\
\hline$(1,2)$ & 8 & 8 & 1 & 69 \\
\hline \hline$(2,2)$ & 2 & 2 & 2 & 14 \\
\hline$(2,3)$ & 4 & 4 & 4 & 42 \\
\hline
\end{tabular}

TABLE II: The number of master integrands are given. No symmetry properties of the integrals are taken into account. Only planar topologies of the master integrals with a central rung are considered. The topologies are specified by (legs left, legs right); this refers to the attached legs on the left/right loop and is denoted as well by $(N-1, \tilde{N}-1)$ in the text. The number of master integrands are obtained in our on-shell approach and validated using off-shell IBP vectors. The counts refer to integrals with standard power counting of QCD like theories but are expected to hold as well beyond this (e.g. gravity theories). For completeness, the total number of irreducible tensor insertions for the given topologies is displayed. The numbers hold for QCD-like power counting. The asterisk ${ }^{*}$ ' marks topologies with bubble diagrams on internal lines which have doubled propagators to start with.

pleteness of the IBP vectors given. Actually, even more directly the count confirms that we have a complete set of surface terms. Here complete refers to the property that the master integrands are distinguishable by unitarity cuts.

The number of integrands is consistent with partially available results [48, 52, 59]. Comparing the number of master integrals is less simple, given that the results for integrals and integrands can differ (due to symmetry properties). Nevertheless, we find the results consistent with available results. 


\section{SUMMARY AND FUTURE DIRECTIONS}

We have discussed new methods and results which are required for multi-loop matrix element generators based on a numerical unitarity approach. The methods form a synthesis of established techniques to obtain integral relations (or surface terms) and the unitarity approach. Interesting connections have been exposed between the classification of loop integrands, special IBP relations [49] and topological properties of unitarity-cut phase spaces.

We presented a number of results: First of all, we constructed a complete set of off-shell integral relations (surface terms) (IV.30) for the planar two-loop topologies. These relations are obtained from a new type of horizontal IBP vectors. We show that their associated IBP relations hold as well if propagator powers are simply changed (section IVD). In addition, as an intermediate result we computed the number of master integrands which is related to a topological property of the unitarity-cut phase spaces.

Further results include the geometric interpretation of IBP vectors and convenient coordinates to identify irreducible tensor insertions (section ЏIF). Moreover, we exposed an important link between on-shell and off-shell integrals of loop amplitudes (section ПII), and identify a useful Lie-algebra structure in the construction (sections IV B, IVC and IVF).

We performed a number of checks. The results for the number of master integrands are consistent with the partially available results [48, 52, 59]. Using the identical input, we count irreducible numerator tensors. The counts match results from earlier constructions of loop integrands [42 44, 61]. We obtained surface terms in two distinct ways; an explicit construction using a generating set of IBP vectors as well as a combinatorial, on-shell approach. The fact that the results agree demonstrates that a complete set of surface terms was obtained. This implies as well that the classification of the generating IBP vectors is complete for the generic planar integrals.

An number of formal questions have to be addressed for computing multi-loop amplitudes based on the presented methods. First of all, it will be important to verify that the provided IBP vectors suffice for integral topologies with vanishing external masses. This is plausible from factorisation properties but needs to be checked in detail. Second, it will be interesting to understand the role of horizontal IBP vectors in higher-loop computations. These vectors are an off-shell continuation of the tangent vectors of the unitarity-cut phase spaces and should continue to be the core structure for the construction of multi-loop inte- 
grands. Third, we exposed a Lie-algebra structure, given that the IBP vectors are generators of rotations. It will be useful to exploit this to simplify computations further (e.g. reduce state sums). Furthermore, with generic expressions given, one may study the integrands' dependence on external momenta (e.g. obtain differential equations for master integrals). Finally, interesting extensions include as well the application of the surface terms to the computation of real pieces and subtraction terms.

The best combination of analytical and numerical methods for multi-loop computations is not obvious in the moment, however, we are motivated by the one-loop successes of the unitarity method to aim for analogous strategy for multi-loop computations. The computation of NNLO QCD cross sections is a very challenging task given that so many pieces have to be controlled and combined. We hope that the methods discussed in this article can contribute to new precision predictions for the LHC experiments on the long run.

\section{Acknowledgments}

I would like to thank S. Abreu, Z. Bern, A. Brandhuber, F. Febres Cordero, M. Jaquier, K. Larsen, B. Page, F. Schlenk and M. Zeng for many helpful discussions and comments. Furthermore, I would like to thank L. Dixon, D. Forde, D. Kosower, D. Maître and K. Ozeren for many inspiring discussions on related topics. This work is supported by a Marie Skłodowska-Curie Action Career-Integration Grant PCIG12-GA-2012-334228 of the European Union and by the Juniorprofessor Program of Ministry of Science, Research and the Arts of the state of Baden-Württemberg, Germany.

\section{Appendix A: Technical construction of algebraic forms}

We can relate the problem of constructing differential forms on smooth algebraic surfaces to reducing an affine coordinate ring by an ideal. We have already encountered the reduction of a polynomial ring by an ideal when constructing irreducible numerators (section ஹIF): to this end we write down all polynomials of the internal $\alpha$-variables and use the on-shell relations $c=\tilde{c}=\hat{c}=0$ to identify equivalent polynomials. The construction of forms is related to this. We start with the standard form calculus in the affine space and consider differential forms with polynomial coefficient functions. When we pull back the forms to the 
maximal-cut phase spaces, initially independent forms become linearly dependent. In order to reduce the linearly dependent forms we use a technical trick; we collect the basis forms as well as the affine coordinates into an extended coordinate ring (see. 62] Chapter II.8 on Differentials for inspiration),

$$
\text { coordinate ring: } \quad\left\{\alpha^{1}, \alpha^{2}, \ldots, y^{1}=d \alpha^{1}, y^{2}=d \alpha^{2}, \ldots\right\}
$$

When pulled back to the maximal-cut phase space, combinations of the forms vanish, which is expressed by an ideal in the auxiliary space of $\alpha$ and $y$ coordinates,

$$
\text { generators of ideal: }\left\{c, \tilde{c}, \hat{c}, \ldots, d c, d \tilde{c}, d \hat{c}, \ldots, y^{i} y^{j}, \ldots\right\}
$$

Here the differentials of the on-shell relations $d c=\left(\partial_{a} c\right) d \alpha^{a}=\left(\partial_{a} c\right) y^{a}$ give polynomial equations mixing $\alpha$ 's and $y$ 's. Furthermore, the relations $y^{i} y^{j}=0$ constrain us to formdegree one. The set of linearly independent forms is then obtained by reducing polynomials in $\alpha$ 's and $y$ 's which are linear in $y^{b}$ by the ideal.

For forms of generic degree $(n)$ one proceeds analogously. Again one includes all basis forms as coordinates,

$$
\begin{aligned}
\text { coordinate ring: } & \left\{\alpha^{1}, \alpha^{2}, \ldots, y^{\left[i_{1}, \ldots, i_{n}\right]}=d \alpha^{\left[i_{1}, i_{2}, \ldots, i_{n}\right]}, \ldots\right\} \\
& \text { with } \quad d \alpha^{\left[i_{1}, i_{2}, \ldots, i_{n}\right]}:=d \alpha^{i_{1}} \wedge \ldots \wedge d \alpha^{i_{n}},
\end{aligned}
$$

and defines relations (an ideal) by the original on-shell relations, their derivatives as well as squares of the differentials,

$$
\begin{aligned}
\text { generators of ideal: } \quad & \left\{c, \tilde{c}, \ldots, d c \wedge d \alpha^{\left[i_{1}, i_{2}, \ldots i_{n-1}\right]}, \ldots, d \tilde{c} \wedge d \alpha^{\left[i_{1}, i_{2}, \ldots i_{n-1}\right]}, \ldots\right. \\
& \left.y^{\left[i_{1}, i_{2}, \ldots, i_{n}\right]} y^{\left[j_{1}, i_{2}, \ldots, j_{n}\right]}, \ldots\right\}
\end{aligned}
$$

where the expressions $d c \wedge d \alpha^{\left[i_{1}, i_{2}, \ldots i_{n-1}\right]}$ have to be written as linear expressions in the coordinates $y^{\left[i_{1}, \ldots i_{n}\right]}$,

$$
d c_{j} \wedge d \alpha^{\left[i_{1}, i_{2}, \ldots, i_{n-1}\right]}=\partial_{b} c_{j} d \alpha^{b} \wedge d \alpha^{\left[i_{1}, i_{2}, \ldots, i_{n-1}\right]}=\left(\partial_{b} c_{j}\right) y^{\left[b, i_{1}, i_{2}, \ldots, i_{n-1}\right]} .
$$

Thus we can start with the set of $n$-forms with polynomial coefficient functions and then use the polynomial reduction procedures to obtain independent $n$-forms on a smooth algebraic variety. The reduction of exact forms can be done in a similar way, where one first generates 
exact forms and then interprets them as polynomials in the auxiliary coordinate ring. The reductions steps work as described above.

[1] S. Catani, L. Cieri, D. de Florian, G. Ferrera and M. Grazzini, "Diphoton production at hadron colliders: a fully-differential QCD calculation at NNLO," Phys. Rev. Lett. 108 (2012) 072001 [arXiv:1110.2375 [hep-ph]].

[2] M. Czakon, P. Fiedler and A. Mitov, "Resolving the Tevatron Top Quark Forward-Backward Asymmetry Puzzle: Fully Differential Next-to-Next-to-Leading-Order Calculation," Phys. Rev. Lett. 115 (2015) 5, 052001 arXiv:1411.3007 [hep-ph]].

[3] T. Gehrmann, M. Grazzini, S. Kallweit, P. Maierhfer, A. von Manteuffel, S. Pozzorini, D. Rathlev and L. Tancredi, " $W^{+} W^{-}$Production at Hadron Colliders in Next to Next to Leading Order QCD," Phys. Rev. Lett. 113 (2014) 21, 212001 [arXiv:1408.5243 [hep-ph]]; F. Cascioli et al., "ZZ production at hadron colliders in NNLO QCD," Phys. Lett. B 735 (2014) 311 arXiv:1405.2219 [hep-ph]].

[4] M. Grazzini, S. Kallweit, D. Rathlev and A. Torre, " $Z \gamma$ production at hadron colliders in NNLO QCD," Phys. Lett. B 731 (2014) 204 [arXiv:1309.7000 [hep-ph]].

[5] R. Boughezal, C. Focke, X. Liu and F. Petriello, " $W$-boson production in association with a jet at next-to-next-to-leading order in perturbative QCD," Phys. Rev. Lett. 115 (2015) 6, 062002 arXiv:1504.02131 [hep-ph]]; A. Gehrmann-De Ridder, T. Gehrmann, E. W. N. Glover, A. Huss and T. A. Morgan, "Precise QCD predictions for the production of a Z boson in association with a hadronic jet," arXiv:1507.02850 [hep-ph].

[6] X. Chen, T. Gehrmann, E. W. N. Glover and M. Jaquier, "Precise QCD predictions for the production of Higgs + jet final states," Phys. Lett. B 740 (2015) 147 arXiv:1408.5325 [hepph]]; R. Boughezal, C. Focke, W. Giele, X. Liu and F. Petriello, "Higgs boson production in association with a jet at NNLO using jettiness subtraction," Phys. Lett. B 748 (2015) 5 arXiv:1505.03893 [hep-ph]]; R. Boughezal, F. Caola, K. Melnikov, F. Petriello and M. Schulze, "Higgs boson production in association with a jet at next-to-next-to-leading order," Phys. Rev. Lett. 115 (2015) 8, 082003 arXiv:1504.07922 [hep-ph]].

[7] C. Anastasiou, C. Duhr, F. Dulat, F. Herzog and B. Mistlberger, "Higgs Boson Gluon-Fusion Production in QCD at Three Loops," Phys. Rev. Lett. 114 (2015) 212001 arXiv:1503.06056 
[hep-ph]].

[8] K. Arnold et al., "VBFNLO: A Parton level Monte Carlo for processes with electroweak bosons," Comput. Phys. Commun. 180 (2009) 1661 [arXiv:0811.4559 [hep-ph]].

[9] C. F. Berger, Z. Bern, L. J. Dixon, F. Febres Cordero, D. Forde, H. Ita, D. A. Kosower and D. Maitre, "An Automated Implementation of On-Shell Methods for One-Loop Amplitudes," Phys. Rev. D 78 (2008) 036003 [arXiv:0803.4180 [hep-ph]].

[10] S. Badger, B. Biedermann and P. Uwer, "NGluon: A Package to Calculate One-loop Multigluon Amplitudes," Comput. Phys. Commun. 182 (2011) 1674 [arXiv:1011.2900 [hep-ph]].

[11] V. Hirschi, R. Frederix, S. Frixione, M. V. Garzelli, F. Maltoni and R. Pittau, "Automation of one-loop QCD corrections," JHEP 1105 (2011) 044 [arXiv:1103.0621 [hep-ph]].

[12] G. Bevilacqua, M. Czakon, M. V. Garzelli, A. van Hameren, A. Kardos, C. G. Papadopoulos, R. Pittau and M. Worek, "Helac-nlo," Comput. Phys. Commun. 184 (2013) 986 arXiv:1110.1499 [hep-ph]].

[13] G. Cullen, N. Greiner, G. Heinrich, G. Luisoni, P. Mastrolia, G. Ossola, T. Reiter and F. Tramontano, "Automated One-Loop Calculations with GoSam," Eur. Phys. J. C 72 (2012) 1889 arXiv:1111.2034 [hep-ph]]; G. Cullen et al., "GOSAM-2.0: a tool for automated one-loop calculations within the Standard Model and beyond," Eur. Phys. J. C 74 (2014) 8, 3001 arXiv:1404.7096 [hep-ph]].

[14] F. Cascioli, P. Maierhofer and S. Pozzorini, "Scattering Amplitudes with Open Loops," Phys. Rev. Lett. 108 (2012) 111601 arXiv:1111.5206 [hep-ph]].

[15] S. Actis, A. Denner, L. Hofer, A. Scharf and S. Uccirati, "Recursive generation of one-loop amplitudes in the Standard Model," JHEP 1304 (2013) 037 arXiv:1211.6316 [hep-ph]].

[16] A. Denner and S. Dittmaier, "Reduction schemes for one-loop tensor integrals," Nucl. Phys. B 734 (2006) 62 [hep-ph/0509141].

[17] Z. Bern, L. J. Dixon, D. C. Dunbar and D. A. Kosower, "One loop n point gauge theory amplitudes, unitarity and collinear limits," Nucl. Phys. B 425 (1994) 217 hep-ph/9403226].

[18] Z. Bern, L. J. Dixon, D. C. Dunbar and D. A. Kosower, "Fusing gauge theory tree amplitudes into loop amplitudes," Nucl. Phys. B 435 (1995) 59 [hep-ph/9409265].

[19] R. Britto, F. Cachazo and B. Feng, "Generalized unitarity and one-loop amplitudes in N=4 super-Yang-Mills," Nucl. Phys. B 725 (2005) 275 hep-th/0412103.

[20] A. Brandhuber, S. McNamara, B. J. Spence and G. Travaglini, "Loop amplitudes in pure Yang- 
Mills from generalised unitarity," JHEP 0510 (2005) 011 hep-th/0506068; S. D. Badger, "Direct Extraction Of One Loop Rational Terms," JHEP 0901 (2009) 049 [arXiv:0806.4600 [hep-ph]]. D. Forde, "Direct extraction of one-loop integral coefficients," Phys. Rev. D 75 (2007) 125019 arXiv:0704.1835 [hep-ph]].

[21] G. Ossola, C. G. Papadopoulos and R. Pittau, "Reducing full one-loop amplitudes to scalar integrals at the integrand level," Nucl. Phys. B 763 (2007) 147 hep-ph/0609007.

[22] R. K. Ellis, W. T. Giele and Z. Kunszt, "A Numerical Unitarity Formalism for Evaluating One-Loop Amplitudes," JHEP 0803 (2008) 003 [arXiv:0708.2398 [hep-ph]]; W. T. Giele, Z. Kunszt and K. Melnikov, "Full one-loop amplitudes from tree amplitudes," JHEP 0804 (2008) 049 [arXiv:0801.2237 [hep-ph]]. R. K. Ellis, W. T. Giele, Z. Kunszt and K. Melnikov, "Masses, fermions and generalized D-dimensional unitarity," Nucl. Phys. B 822 (2009) 270 arXiv:0806.3467 [hep-ph]];

[23] K. G. Chetyrkin and F. V. Tkachov, "Integration by Parts: The Algorithm to Calculate beta Functions in 4 Loops," Nucl. Phys. B 192 (1981) 159;

[24] A. von Manteuffel and C. Studerus, "Reduze 2 - Distributed Feynman Integral Reduction," arXiv:1201.4330 [hep-ph]. C. Studerus, "Reduze-Feynman Integral Reduction in C++," Comput. Phys. Commun. 181 (2010) 1293 [arXiv:0912.2546 [physics.comp-ph]]. A. von Manteuffel and R. M. Schabinger, "A novel approach to integration by parts reduction," Phys. Lett. B $\mathbf{7 4 4}(2015) 101$ arXiv:1406.4513 [hep-ph]].

[25] C. Anastasiou and A. Lazopoulos, "Automatic integral reduction for higher order perturbative calculations," JHEP 0407 (2004) 046 hep-ph/0404258.

[26] A. V. Smirnov and V. A. Smirnov, "FIRE4, LiteRed and accompanying tools to solve integration by parts relations," Comput. Phys. Commun. 184 (2013) 2820 arXiv:1302.5885 [hepph]]; A. V. Smirnov and V. A. Smirnov, "FIRE4, LiteRed and accompanying tools to solve integration by parts relations," Comput. Phys. Commun. 184 (2013) 2820 arXiv:1302.5885 [hep-ph]];

[27] A. V. Smirnov, "FIRE5: a C++ implementation of Feynman Integral REduction," Comput. Phys. Commun. 189 (2014) 182 arXiv:1408.2372 [hep-ph]].

[28] R. N. Lee, "LiteRed 1.4: a powerful tool for reduction of multiloop integrals," J. Phys. Conf. Ser. 523 (2014) 012059 [arXiv:1310.1145 [hep-ph]].

[29] S. Laporta, "High precision calculation of multiloop Feynman integrals by difference equa- 
tions," Int. J. Mod. Phys. A 15 (2000) 5087 [hep-ph/0102033].

[30] R. K. Ellis, W. T. Giele, Z. Kunszt, K. Melnikov and G. Zanderighi, JHEP 0901 (2009) 012 arXiv:0810.2762 [hep-ph]].

[31] C. F. Berger et al., "Precise Predictions for $W+3$ Jet Production at Hadron Colliders," Phys. Rev. Lett. 102 (2009) 222001 arXiv:0902.2760 [hep-ph]].

[32] R. K. Ellis, K. Melnikov and G. Zanderighi, "W+3 jet production at the Tevatron," Phys. Rev. D 80 (2009) 094002 [arXiv:0906.1445 [hep-ph]].

[33] C. F. Berger et al., "Precise Predictions for W + 4 Jet Production at the Large Hadron Collider," Phys. Rev. Lett. 106 (2011) 092001 [arXiv:1009.2338 [hep-ph]].

[34] Z. Bern, L. J. Dixon, F. Febres Cordero, S. Hche, H. Ita, D. A. Kosower, D. Matre and K. J. Ozeren, "Next-to-Leading Order $W+5$-Jet Production at the LHC," Phys. Rev. D 88 (2013) 1, 014025 [arXiv:1304.1253 [hep-ph]].

[35] S. Badger, B. Biedermann, P. Uwer and V. Yundin, "Next-to-leading order QCD corrections to five jet production at the LHC," Phys. Rev. D 89 (2014) 3, 034019 [arXiv:1309.6585 [hep-ph]].

[36] Z. Bern, L. J. Dixon and D. A. Kosower, "A Two loop four gluon helicity amplitude in QCD," JHEP 0001 (2000) 027 hep-ph/0001001]. Z. Bern, A. De Freitas and L. J. Dixon, "Two loop helicity amplitudes for gluon-gluon scattering in QCD and supersymmetric Yang-Mills theory," JHEP 0203 (2002) 018 [hep-ph/0201161]. Z. Bern, A. De Freitas and L. J. Dixon, "Two loop helicity amplitudes for quark gluon scattering in QCD and gluino gluon scattering in supersymmetric Yang-Mills theory," JHEP 0306 (2003) 028 [JHEP 1404 (2014) 112] hep-ph/0304168.

[37] Z. Bern, L. J. Dixon, D. C. Dunbar, M. Perelstein and J. S. Rozowsky, "On the relationship between Yang-Mills theory and gravity and its implication for ultraviolet divergences," Nucl. Phys. B 530, 401 (1998) hep-th/9802162;

[38] Z. Bern, J. J. Carrasco, L. J. Dixon, H. Johansson and R. Roiban, "The Ultraviolet Behavior of N=8 Supergravity at Four Loops," Phys. Rev. Lett. 103 (2009) 081301 arXiv:0905.2326 [hep-th]].

[39] Z. Bern, L. J. Dixon and V. A. Smirnov, "Iteration of planar amplitudes in maximally supersymmetric Yang-Mills theory at three loops and beyond," Phys. Rev. D 72 (2005) 085001 hep-th/0505205.

[40] N. Arkani-Hamed, J. L. Bourjaily, F. Cachazo, S. Caron-Huot and J. Trnka, "The All- 
Loop Integrand For Scattering Amplitudes in Planar N=4 SYM," JHEP 1101 (2011) 041 arXiv:1008.2958 [hep-th]]; J. L. Bourjaily and J. Trnka, "Local Integrand Representations of All Two-Loop Amplitudes in Planar SYM," JHEP 1508 (2015) 119 arXiv:1505.05886 [hep-th]].

[41] S. Badger, H. Frellesvig and Y. Zhang, "A Two-Loop Five-Gluon Helicity Amplitude in QCD," JHEP 1312 (2013) 045 [arXiv:1310.1051 [hep-ph]]; S. Badger, G. Mogull, A. Ochirov and D. O'Connell, "A Complete Two-Loop, Five-Gluon Helicity Amplitude in Yang-Mills Theory,” arXiv:1507.08797 [hep-ph].

[42] P. Mastrolia and G. Ossola, "On the Integrand-Reduction Method for Two-Loop Scattering Amplitudes," JHEP 1111 (2011) 014 [arXiv:1107.6041 [hep-ph]].

[43] S. Badger, H. Frellesvig and Y. Zhang, "Hepta-Cuts of Two-Loop Scattering Amplitudes," JHEP 1204 (2012) 055 [arXiv:1202.2019 [hep-ph]].

[44] Y. Zhang, "Integrand-Level Reduction of Loop Amplitudes by Computational Algebraic Geometry Methods," JHEP 1209 (2012) 042 [arXiv:1205.5707 [hep-ph]].

[45] E. I. Buchbinder and F. Cachazo, "Two-loop amplitudes of gluons and octa-cuts in N=4 super Yang-Mills," JHEP 0511 (2005) 036 hep-th/0506126; F. Cachazo, "Sharpening The Leading Singularity," arXiv:0803.1988 [hep-th]; Z. Bern, J. J. M. Carrasco, H. Johansson and D. A. Kosower, "Maximally supersymmetric planar Yang-Mills amplitudes at five loops," Phys. Rev. D 76 (2007) 125020 arXiv:0705.1864 [hep-th]].

[46] D. A. Kosower and K. J. Larsen, "Maximal Unitarity at Two Loops," Phys. Rev. D 85 (2012) 045017 arXiv:1108.1180 [hep-th]];

[47] S. Caron-Huot and K. J. Larsen, "Uniqueness of two-loop master contours," JHEP 1210 (2012) 026 [arXiv:1205.0801 [hep-ph]].

[48] A. Georgoudis and Y. Zhang, "Two-loop Integral Reduction from Elliptic and Hyperelliptic Curves," arXiv:1507.06310 [hep-th].

[49] J. Gluza, K. Kajda and D. A. Kosower, "Towards a Basis for Planar Two-Loop Integrals," Phys. Rev. D 83, 045012 (2011) arXiv:1009.0472 [hep-th]].

[50] R. M. Schabinger, "A New Algorithm For The Generation Of Unitarity-Compatible Integration By Parts Relations," JHEP 1201 (2012) 077 [arXiv:1111.4220 [hep-ph]].

[51] Y. Zhang, "Integration-by-parts identities from the viewpoint of differential geometry," arXiv:1408.4004 [hep-th]. 
[52] M. Søgaard and Y. Zhang, "Elliptic Functions and Maximal Unitarity," Phys. Rev. D 91, no. 8, 081701 (2015) [arXiv:1412.5577 [hep-th]].

[53] A. Georgoudis and Y. Zhang, "Two-loop Integral Reduction from Elliptic and Hyperelliptic Curves," arXiv:1507.06310 [hep-th].

[54] W. L. van Neerven and J. A. M. Vermaseren, "Large Loop Integrals," Phys. Lett. B 137 (1984) 241.

[55] G. 't Hooft and M. J. G. Veltman, "Scalar One Loop Integrals," Nucl. Phys. B 153, 365 (1979); G. J. van Oldenborgh and J. A. M. Vermaseren, "New Algorithms for One Loop Integrals," Z. Phys. C 46, 425 (1990); W. Beenakker and A. Denner, "Infrared Divergent Scalar Box Integrals With Applications in the Electroweak Standard Model," Nucl. Phys. B 338, 349 (1990); A. Denner, U. Nierste and R. Scharf, "A Compact expression for the scalar one loop four point function," Nucl. Phys. B 367, 637 (1991); Z. Bern, L. J. Dixon and D. A. Kosower, "Dimensionally regulated pentagon integrals," Nucl. Phys. B 412 (1994) 751 hep-ph/9306240]; T. Hahn and M. Pérez-Victoria, "Automatized one-loop calculations in four and D dimensions," Comput. Phys. Commun. 118, 153 (1999) hep-ph/9807565; R. K. Ellis and G. Zanderighi, "Scalar one-loop integrals for QCD," JHEP 0802, 002 (2008) [0712.1851 [hep-ph]].

[56] H. Ita, "Susy Theories and QCD: Numerical Approaches," J. Phys. A 44 (2011) 454005 arXiv:1109.6527 [hep-th]].

[57] H.J. Dirschmid, "Tensoren und Felder,", Springer-Verlag/Vienna (1996); M. Nakahara, "Geometry, topology and physics," Boca Raton, USA: Taylor \& Francis (2003) 573 p

[58] O. V. Tarasov, "Generalized recurrence relations for two loop propagator integrals with arbitrary masses," Nucl. Phys. B 502 (1997) 455 [hep-ph/9703319].

[59] R. M. Schabinger (presenter), A. von Manteuffel, "The Two-Loop Analog of the PassarinoVeltman Result And Beyond", PoS RADCOR 2013 (2013).

[60] S. Wolfram, The Mathematica book, 5th edition, Wolfram Media, Inc., 2003.

[61] B. Feng and R. Huang, "The classification of two-loop integrand basis in pure four-dimension," JHEP 1302 (2013) 117 [arXiv:1209.3747[ [hep-ph]].

[62] R.Hartshorne, "Algebraic Geometry,", Springer-Verlag/New York (1977)

[63] R. J. Eden, P. V. Landshoff, D. I. Olive, J. C. Polkinghorne, "The Analytic S-Matrix," Cambridge University Press (1966) 